\title{
WestVirginiaUniversity
}

THE RESEARCH REPOSITORY @ WVU

Graduate Theses, Dissertations, and Problem Reports

2008

\section{Cycles in graph theory and matroids}

Ju Zhou

West Virginia University

Follow this and additional works at: https://researchrepository.wvu.edu/etd

\section{Recommended Citation}

Zhou, Ju, "Cycles in graph theory and matroids" (2008). Graduate Theses, Dissertations, and Problem Reports. 2720.

https://researchrepository.wvu.edu/etd/2720

This Dissertation is protected by copyright and/or related rights. It has been brought to you by the The Research Repository @ WVU with permission from the rights-holder(s). You are free to use this Dissertation in any way that is permitted by the copyright and related rights legislation that applies to your use. For other uses you must obtain permission from the rights-holder(s) directly, unless additional rights are indicated by a Creative Commons license in the record and/ or on the work itself. This Dissertation has been accepted for inclusion in WVU Graduate Theses, Dissertations, and Problem Reports collection by an authorized administrator of The Research Repository @ WVU.

For more information, please contact researchrepository@mail.wvu.edu. 


\title{
Cycles in Graph Theory and Matroids
}

\author{
Ju Zhou \\ Dissertation submitted to the \\ Eberly College of Arts and Sciences \\ at West Virginia University \\ in partial fulfillment of the requirements \\ for the degree of \\ Doctor of Philosophy \\ in \\ Mathematics
Hong-Jian Lai, Ph.D., Chair
John Goldwasser, Ph.D.
K. Subramani, Ph.D.
Jerzy Wojciechowski, Ph.D.
Cun-Quan Zhang, Ph.D. \\ Department of Mathematics \\ Morgantown, West Virginia \\ 2008
}

Keywords: Hamiltonian, Hamiltonian-Connected, s-Hamiltonian, s-Hamiltonian Connected, Eulerian, Supereulerian, Line Graph, Claw-Free Graph 


\begin{abstract}
A circuit is a connected 2-regular graph. A cycle is a graph such that the degree of each vertex is even. A graph $G$ is Hamiltonian if it has a spanning circuit, and Hamiltonianconnected if for every pair of distinct vertices $u, v \in V(G), G$ has a spanning $(u, v)$ path. A graph $G$ is $s$-Hamiltonian if for any $S \subseteq V(G)$ of order at most $s, G-S$ has a Hamiltonian-circuit, and $s$-Hamiltonian connected if for any $S \subseteq V(G)$ of order at most $s, G-S$ is Hamiltonian-connected. In this dissertation, we investigated sufficient conditions for Hamiltonian and Hamiltonian related properties in a graph or in a line graph. In particular, we obtained sufficient conditions in terms of connectivity only for a line graph to be Hamiltonian, and sufficient conditions in terms of degree for a graph to be s-Hamiltonian and s-Hamiltonian connected.

A cycle $C$ of $G$ is a spanning eulerian subgraph of $G$ if $C$ is connected and spanning. A graph $G$ is supereulerian if $G$ contains a spanning eulerian subgraph. If $G$ has vertices $v_{1}, v_{2}, \cdots, v_{n}$, the sequence $\left(d\left(v_{1}\right), d\left(v_{2}\right), \cdots, d\left(v_{n}\right)\right)$ is called a degree sequence of $G$. A sequence $d=\left(d_{1}, d_{2}, \cdots, d_{n}\right)$ is graphic if there is a simple graph $G$ with degree sequence $d$. Furthermore, $G$ is called a realization of $d$. A sequence $d \in \mathcal{G}$ is line-hamiltonian if $d$ has a realization $G$ such that $L(G)$ is hamiltonian. In this dissertation, we obtained sufficient conditions for a graphic degree sequence to have a supereulerian realization or to be line hamiltonian.

In 1960, Erdös and Pósa characterized the graphs $G$ which do not have two edgedisjoint circuits. In this dissertation, we successfully extended the results to regular matroids and characterized the regular matroids which do not have two disjoint circuits.
\end{abstract}

\title{
Cycles in Graph Theory and Matroids
}

\section{Ju Zhou}




\section{Acknowledgements}

First, I'd like to express my sincere thanks to my supervisor, Dr. Hong-Jian Lai, for his guidance, encouragement, help and support during all these years. It has been a great pleasure to work under his supervision and I am lucky enough to have had a chance to learn from him. What I learned from him will benefit me all my life.

Second, I'd also like to thank my committee members: Dr. John Goldwasser, Dr. Hong-Jian Lai, Dr. K. Subramani, Dr. Jerzy Wojciechowski, and Dr. Cun-Quan Zhang, for their help and support during my studies.

Third, I want to take this chance to thank my previous supervisor Dr. Jinjiang Yuan in China for his guidance during his supervision and his continuous care and encouragement about my progress after that.

Fourth, I'd also like to thank my parents for their life-long moral support, their expectations and encouragement and my husband for his care and love. It is the company of their love which makes me feel not lonely and full of energy on my way to pursuing knowledge.

And finally, I would like to thank the Department of Mathematics and Eberly College of Arts and Sciences at West Virginia University for providing me with an excellent study environment and continual support during my yeas as a graduate student. 


\section{Contents}

1 Introduction 1

1.1 Notation and Terminology . . . . . . . . . . . . . . . . . 1

1.2 Road Map . . . . . . . . . . . . . . . . . . . . 4

1.3 Main Results . . . . . . . . . . . . . . . . . . . 4

1.3.1 Partial Results Towards Thomassen Conjecture . . . . . . . . . . . 4

1.3.2 s-Hamiltonian and s-Hamiltonian Connected . . . . . . . . . . 6

1.3.3 Degree Sequence and Supereulerian Graphs _. . . . . . . . . . . 7

1.3.4 Regular Matroids without Disjoint Circuits . . . . . . . . . . . 8

2 Partial Result towards Thomassen Conjecture 10

2.1 The Problem and the Main Results . . . . . . . . . . . . . . . . . 10

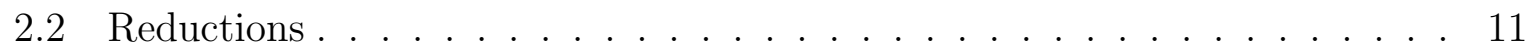

2.3 Proof of Theorem $2.1 .5 \ldots \ldots \ldots \ldots \ldots \ldots$

3 s-Hamiltonian and s-Hamiltonian Connected 16 
3.1 The Problem and the Main Results . . . . . . . . . . . . . . . . 16

3.2 Proof of Theorem $3.1 .7 \ldots \ldots \ldots \ldots \ldots$

3.3 Proof of Theorem $3.1 .8 \ldots \ldots \ldots \ldots \ldots \ldots \ldots$

4 Degree Sequence and Supereulerian Graphs $\quad 25$

4.1 The Problem and the Main Results . . . . . . . . . . . . . . . . 25

4.2 Collapsible Sequences . . . . . . . . . . . . . . . . . . . . . . 26

4.3 Supereulerian Sequence and Hamiltonian Line Graph . . . . . . . . . . . . 30

5 Regular Matroids without Disjoint Circuits $\quad 36$

5.1 The Problem and the Main Results . . . . . . . . . . . . . . 36

5.2 Proof of the Main Results . . . . . . . . . . . . . . . . . . 38 


\section{DEDICATION}

To

my parents Guoqiang Zhou and Huage Jia, my siblings Peng Zhou and Pan Zhou

and

my husband Taoye Zhang 


\section{Chapter 1}

\section{Introduction}

\subsection{Notation and Terminology}

We use [2] for notations and terminology in graph theory not defined here, and consider finite loopless connected graphs. For a graph $G$, we use $V(G), E(G), \delta(G)$ and $\alpha(G)$ to denote its vertex set, edge set, minimal degree and independence number, respectively. In particular, $\kappa(G)$ and $\kappa^{\prime}(G)$ represent the connectivity and edge-connectivity of a graph $G$.

A graph is trivial if it contains no edges. A vertex cut $X$ of $G$ is essential if $G-X$ has at least two nontrivial components. For an integer $k>0$, a graph $G$ is essentially $k$-connected if $G$ does not have an essential cut $X$ with $|X|<k$. An edge cut $Y$ of $G$ is essential if $G-Y$ has at least two nontrivial components. For an integer $k>0$, a graph $G$ is essentially $k$-edge-connected if $G$ does not have an essential edge cut $Y$ with $|Y|<k$.

The line graph of a graph $G$, denoted by $L(G)$, has $E(G)$ as its vertex set, where two vertices in $L(G)$ are adjacent if and only if the corresponding edges in $G$ have at least one vertex in common. From the definition of a line graph, if $L(G)$ is not a complete graph, then a subset $X \subseteq V(L(G))$ is a vertex cut of $L(G)$ if and only if $X$ is an essential edge cut of $G$. 
A circuit is a connected 2-regular graph. A cycle is a graph such that the degree of each vertex is even. A graph $G$ is Hamiltonian if it has a spanning circuit, and Hamiltonianconnected if for every pair of distinct vertices $u, v \in V(G), G$ has a spanning $(u, v)$-path. A graph $G$ is $s$-Hamiltonian if for any $S \subseteq V(G)$ of order at most $s, G-S$ has a Hamiltonian-circuit, and $s$-Hamiltonian connected if for any $S \subseteq V(G)$ of order at most $s, G-S$ is Hamiltonian-connected. A cycle $C$ of $G$ is a spanning eulerian subgraph of $G$ if $C$ is connected and spanning. A graph $G$ is supereulerian if $G$ contains a spanning eulerian subgraph.

The contraction $G / X$ is the graph obtained from $G$ be identifying the two ends of each edge in $X$ and then deleting the resulting loops. When $X=\{e\}$, we also use $G / e$ for $G /\{e\}$. For an integer $i>0$, define

$$
D_{i}(G)=\left\{v \in V(G): \operatorname{deg}_{G}(v)=i\right\} .
$$

For any $v \in V(G)$, define

$$
E_{G}(v)=\{e \in E(G): e \text { is incident with } v \text { in } G\} .
$$

Catlin in [3] introduced collapsible graphs. A graph $G$ is collapsible if for any subset $R \subseteq V(G)$ with $|R| \equiv 0(\bmod 2), G$ has a spanning connected subgraph $H_{R}$ such that $O\left(H_{R}\right)=R$. Note that when $R=\emptyset$, a spanning connected subgraph $H$ with $O(H)=\emptyset$ is a spanning Eulerian subgraph of $G$. Thus every collapsible graph is supereulerian. Catlin ([3]) showed that any graph $G$ has a unique subgraph $H$ such that every component of $H$ is a maximally collapsible subgraph of $G$ and every nontrivial collapsible subgraph of $G$ is contained in a component of $H$. The contraction $G / H$ is called the reduction of $G$. A graph $G$ is reduced if it is the reduction of itself.

If $G$ has vertices $v_{1}, v_{2}, \cdots, v_{n}$, the sequence $\left(d\left(v_{1}\right), d\left(v_{2}\right), \cdots, d\left(v_{n}\right)\right)$ is called a degree sequence of $G$. A sequence $d=\left(d_{1}, d_{2}, \cdots, d_{n}\right)$ is nonincreasing if $d_{1} \geq d_{2} \geq \cdots d_{n}$. A sequence $d=\left(d_{1}, d_{2}, \cdots, d_{n}\right)$ is graphic if there is a simple graph $G$ with degree sequence $d$. Furthermore, $G$ is called a realization of $d$. Let $\mathcal{G}$ be the set of all graphic degree sequences. A sequence $d \in \mathcal{G}$ is line-hamiltonian if $d$ has a realization $G$ such that $L(G)$ is hamiltonian. A sequence $d=\left(d_{1}, d_{2}, \cdots, d_{n}\right)$ is collapsible if $d$ has a simple collapsible realization. 
Let $H_{1}, H_{2}$ be subgraphs of a graph $G$. Then $H_{1} \cup H_{2}$ is a subgraph of $G$ with vertex set $V\left(H_{1}\right) \cup V\left(H_{2}\right)$ and edge set $E\left(H_{1}\right) \cup E\left(H_{2}\right)$; and $H_{1} \cap H_{2}$ is a subgraph of $G$ with vertex set $V\left(H_{1}\right) \cap V\left(H_{2}\right)$ and edge set $E\left(H_{1}\right) \cap E\left(H_{2}\right)$. If $V_{1}, V_{2}$ are two disjoint subsets of $V(G)$, then $\left[V_{1}, V_{2}\right]_{G}$ denotes the set of edges in $G$ with one end in $V_{1}$ and the other end in $V_{2}$. When the graph $G$ is understood from the context, we also omit the subscript $G$ and write $\left[V_{1}, V_{2}\right]$ for $\left[V_{1}, V_{2}\right]_{G}$. If $H_{1}, H_{2}$ are two vertex disjoint subgraphs of $G$, then we also write $\left[H_{1}, H_{2}\right]$ for $\left[V\left(H_{1}\right), V\left(H_{2}\right)\right]$.

We use Oxley [18] or Welsh [24] for notations and terminology of matroids not defined here. In combinatorics, a matroid is a structure that captures the essence of a notion of independence that generalizes linear independence in vector spaces. One of the most valuable definitions is that in terms of independence. In this definition, a finite matroid $M$ is a pair $(E, \mathcal{I})$, where $E$ is a finite set and $\mathcal{I}$ is a collection of subsets of $E$ (called the independent sets) with the following properties:

(1) The empty set is independent.

(2) Every subset of an independent set is independent.

(3) If $A$ and $B$ are two independent sets and $A$ has more elements than $B$, then there exists an element in $A$ which is not in $B$ and when added to $B$ still gives an independent set.

Besides the vector spaces of linear algebra, a second original source for the theory of matroids is graph theory. Every finite graph (or multigraph) $G$ gives rise to a matroid as follows: take as $E$ the set of all edges in $G$ and consider a set of edges independent if and only if it does not contain a simple circuit. Such an edge set is called a forest in graph theory. This is called the cycle matroid or graphic matroid of $G$; it is usually written $M(G)$. Any matroid that is equivalent to the cycle matroid of a (multi)graph, even if it is not presented in terms of graphs, is called a graphic matroid. The matroids that are graphic have been characterized by Tutte.

A subset of $E$ that is not independent is called dependent. A circuit in a matroid $M$ is a minimal dependent subset of $E$. A cycle in a matroid $M$ is disjoint union of circuits $M$. A matroid is regular if it can be represented by a totally unimodular matrix (a matrix whose square submatrices all have determinants equal to 0,1 , or -1 ). It's not hard to verify that every graphic matroid is regular matroid. 


\subsection{Road Map}

This dissertation consists of 5 chapters. Starting from chapter 2, each chapter will be a study on a specific topic. In Chapter 2, we will investigate the sufficient condition in terms of connectivity for a line graph to be Hamiltonian. In Chapter 3, we will investigate the sufficient conditions in terms of degree for a graph to be $s$-Hamiltonian or $s$-Hamiltonian connected. In Chapter 4, we investigate sufficient conditions for a graphic degree sequence to have a supereulerian realization or to be line hamiltonian. In Chapter 5, we extend the characterization of graphs without two edge-disjoint circuits to the characterization of matroids without two disjoint circuits.

\subsection{Main Results}

There are extensive researches about circuits in graph theory. One part of my work is related to problems in graph theory involving Hamiltonian, Hamiltonian-connected, s-Hamiltonian, S-Hamiltonian connected and supereulerian.

Bill Tutte once said: if a theorem about graphs can be expressed in terms of edges and circuits alone, it probably exemplifies a more general theorem about matroids. The other part of my work is to generalize some known results in graph theory to matroids.

\subsubsection{Partial Results Towards Thomassen Conjecture}

In 1986, Thomassen proposed the following conjecture.

Conjecture 1.3.1 (Thomassen [23]) Every 4-connected line graph is hamiltonian.

A graph that does not have an induced subgraph isomorphic to $K_{1,3}$ is called a clawfree graph. It is well known that every line graph is a claw-free graph. Matthews and Sumner proposed a seemingly stronger conjecture. 
Conjecture 1.3.2 (Matthews and Sumner [16]) Every 4-connected claw-free graph is hamiltonian.

The best result towards these conjectures so far were obtained by Zhan and Ryjáček.

Theorem 1.3.3 (Zhan [25]) Every \%-connected line graph is hamiltonian connected.

Theorem 1.3.4 (Ryjáček [19])

(i) Conjecture 1.1 and Conjecture 1.2 are equivalent.

(ii) Every 7-connected claw-free graph is hamiltonian.

It is well known that the line graph of the graph obtained by subdividing each edge of the Petersen graph exactly once is a 3-connected claw-free graph without a hamiltonian circuit. We consider the following problem: For 3-connected claw-free graphs, can high essential connectivity guarantee the existence of a hamiltonian circuit? This leads us to prove the following Theorem 1.3.5.

Theorem 1.3.5 Every 3-connected, essentially 11-connected line graph is hamiltonian.

Ryjáček [19] introduced the line graph closure of a claw-free graph and used it to show that a claw-free graph $G$ is hamiltonian if and only if its closure $c l(G)$ is hamiltonian, where $c l(G)$ is a line graph. With this argument and using the fact that adding edges will not decrease the connectivity of a graph, the following corollary is obtained.

Corollary 1.3.6 Every 3-connected, essentially 11-connected claw-free graph is hamiltonian.

However, what is the smallest positive integer $k$ such that every 3 -connected, essentially $k$-connected claw-free graph is hamiltonian? This question remains to be answered. Corollary 1.3.6 suggests that $4 \leq k \leq 11$. We fail to construct examples to show that there exists a 3-connected essentially 4-connected non-hamiltonian claw-free graph, and we conjecture that $k=4$. 


\subsection{2 s-Hamiltonian and s-Hamiltonian Connected}

The following sufficient conditions to ensure the existence of a Hamiltonian circuit in a simple graph $G$ of order $n \geq 3$ are well known.

Theorem 1.3.7 (Dirac [6]) If $\delta(G) \geq n / 2$, then $G$ is Hamiltonian.

Theorem 1.3.8 (Ore [17]) If $d(u)+d(v) \geq n$ for each pair of nonadjacent vertices $u, v \in V(G)$, then $G$ is Hamiltonian.

Theorem 1.3.9 (Fan [9]) If $G$ is a 2-connected graph and if $\max \{d(u), d(v)\} \geq n / 2$ for each pair of vertices $u, v \in V(G)$ with $d(u, v)=2$, then $G$ is Hamiltonian.

Theorem 1.3.10 (Chen [4]) If $G$ is a 2-connected graph and if $\max \{d(u), d(v)\} \geq n / 2$ for each pair of vertices $u, v \in V(G)$ with $1 \leq|N(u) \cap N(v)| \leq \alpha(G)-1$, then $G$ is Hamiltonian.

Theorem 1.3.11 (Chen et al [5]) If $G$ is a $k$-connected $(k \geq 2)$ graph and if $\max \{d(v)$ : $v \in I\} \geq n / 2$ for every independent set $I$ of order $k$ such that $I$ has two distinct vertices $x, y$ with $d(x, y)=2$, then $G$ is Hamiltonian.

Zhao et al recently proved Theorem 1.3.12 below, which unified and extended the above theorems.

Theorem 1.3.12 (Zhao et al [14]) If $G$ is a $k$-connected $(k \geq 2)$ graph of order $n$ and if $\max \{d(v): v \in I\} \geq n / 2$ for every independent set $I$ of order $k$ such that $I$ has two distinct vertices $x, y$ with $1 \leq|N(x) \cap N(y)| \leq \alpha(G)-1$, then $G$ is Hamiltonian.

We shall obtain sufficient conditions for $s$-Hamiltonian graphs and $s$-Hamiltonian connected graphs, respectively, as shown below. 
Theorem 1.3.13 Let $k, s$ be two integers with $k \geq s+2$ and $0 \leq s \leq n-3$. If $G$ is a $k$-connected graph of order $n$ and if $\max \{d(v): v \in I\} \geq(n+s) / 2$ for every independent set $I$ of order $k-s$ such that $I$ has two distinct vertices $x, y$ with $1 \leq|N(x) \cap N(y)| \leq$ $\alpha(G)+s-1$, then $G$ is s-Hamiltonian.

Theorem 1.3.14 Let $k, s$ be two integers with $k \geq s+3$ and $0 \leq s \leq n-2$. If $G$ is a k-connected graph of order $n$ and if $\max \{d(v): v \in I\} \geq(n+s+1) / 2$ for every independent set $I$ of order $k-s-1$ such that $I$ has two distinct vertices $x, y$ with $1 \leq$ $|N(x) \cap N(y)| \leq \alpha(G)+s$, then $G$ is s-Hamiltonian connected.

Note that Theorem 1.3.12 is a special case of Theorem 1.3.13 when $s=0$. Applying Theorem 1.3.14 to the case when $s=0$, we get the following corollary.

Corollary 1.3.15 If $G$ is a $k$-connected $(k \geq 3)$ graph of order $n$ and if $\max \{d(v): v \in$ $I\} \geq(n+1) / 2$ for every independent set $I$ of order $k-1$ such that $I$ has two distinct vertices $x, y$ with $1 \leq|N(x) \cap N(y)| \leq \alpha(G)$, then $G$ is Hamiltonian-connected.

\subsubsection{Degree Sequence and Supereulerian Graphs}

In [26], Zhang et al. proved the following theorem.

Theorem 1.3.16 [26] Every bipartite graphic sequence with the minimum degree $\delta \geq 2$ has a realization that admits a nowhere-zero 4-flow.

We first get the following result.

Theorem 1.3.17 If $d=\left(d_{1}, d_{2}, \cdots, d_{n}\right) \in \mathcal{G}$ is a nonincreasing sequences with $d_{n} \geq 2$, then $d$ has a supereulerian realization.

In [12], Jaeger proved the following result. 
Theorem 1.3.18 [12] Every supereulerian graph admits a nowhere-zero 4-flow.

Combining Theorem 4.1.3, we get a result analogous to Theorem 1.3.16.

Theorem 1.3.19 If $d=\left(d_{1}, d_{2}, \cdots, d_{n}\right) \in \mathcal{G}$ is a nonincreasing sequences with $d_{n} \geq 2$, then $d$ has a realization that admits a nowhere-zero 4 -flow.

Furthermore, we get a result about line-hamiltonian sequence as follows.

Theorem 1.3.20 If $d=\left(d_{1}, d_{2}, \cdots, d_{n}\right)$ is a nonincreasing graphic sequence with $n \geq 3$, then the following are equivalent.

(i) d is line-hamiltonian.

(ii) $d \in \mathcal{G}$ and either $d_{1}=n-1$, or

$$
\sum_{d_{i}=1} d_{i} \leq \sum_{d_{j} \geq 2}\left(d_{j}-2\right)
$$

(iii) d has a realization $G$ such that $G-D_{1}(G)$ is supereulerian.

\subsubsection{Regular Matroids without Disjoint Circuits}

In 1960, Erdös and Pósa consider the problem of determining all connected graphs that do not have edge-disjoint circuits. We view the complete graph $K_{3}$ as a plane graph and let $K_{3}^{*}$ denote the geometric dual of the plane graph $K_{3}$.

Theorem 1.3.21 (Erdös and Pósa [8], also see Theorem 3.1, Theorem 3.2 of Bollobás [1]) Let $G$ be a graph with $\delta(G) \geq 3$. The following are equivalent.

(i) $G$ does not have edge-disjoint circuits.

(ii) $G \in\left\{K_{3,3}, K_{3}^{*}, K_{4}\right\}$. 
Since a graph $G$ does not have disjoint circuits if and only if any subdivision of $G$ does not have disjoint circuits, the following corollary follows immediately.

Corollary 1.3.22 (Erdös and Pósa [8], also see Corollary 3.3 of Bollobás [1]) Let G be a simple graph of order $n \geq 3$.

(i) If $|E(G)| \geq n+4$, then $G$ has 2 edge-disjoint circuits.

(ii) The graph $G$ with $|E(G)|=n+3$ does not have edge-disjoint circuits if and only if $G$ can be obtained from a subdivision $G_{0}$ of $K_{3,3}$ by adding a forest and exactly one edge, joining each tree of the forest to $G_{0}$.

Theorem 1.3.21 can be viewed as a result on cosimple graphic matroids. Thus we consider generalizing Theorem 1.3.21 to matroids. Our main results of this note are the following.

Theorem 1.3.23 Let $M$ be a connected cosimple regular matroid. The following are equivalent.

(i) $M$ does not have disjoint circuits.

(ii) $M \in\left\{M\left(K_{3,3}\right)\right\} \cup\left\{M^{*}\left(K_{n}\right), n \geq 3\right\}$.

Corollary 1.3.24 Let $M$ be a regular matroid. Then $M$ has no disjoint circuits if and only if one of the following holds:

(i) $M=U_{m, m}$, for some integer $m>0$, or

(ii) $M$ is a serial extension of a member in $\left\{M\left(K_{3,3}\right), U_{0,1}\right\} \cup\left\{M^{*}\left(K_{n}\right), n \geq 3\right\}$, or

(iii) $M=M_{1} \bigoplus M_{2}$ is the direct sum of two matroids $M_{1}$ and $M_{2}$, where $M_{1}$ is a serial extension of a member in $\left\{M\left(K_{3,3}\right), U_{0,1}\right\} \cup\left\{M^{*}\left(K_{n}\right), n \geq 3\right\}$ and where $M_{2} \cong U_{m, m}$, for some $m=|E(M)|-\left|E\left(M_{1}\right)\right| \geq 1$. 


\section{Chapter 2}

\section{Partial Result towards Thomassen Conjecture}

\subsection{The Problem and the Main Results}

In 1986, Thomassen proposed the following conjecture.

Conjecture 2.1.1 (Thomassen [23]) Every 4-connected line graph is hamiltonian.

A graph that does not have an induced subgraph isomorphic to $K_{1,3}$ is called a clawfree graph. It is well known that every line graph is a claw-free graph. Matthews and Sumner proposed a seemingly stronger conjecture.

Conjecture 2.1.2 (Matthews and Sumner [16]) Every 4-connected claw-free graph is hamiltonian.

The best result towards these conjectures so far were obtained by Zhan and Ryjáček.

Theorem 2.1.3 (Zhan [25]) Every 7-connected line graph is hamiltonian connected. 
Theorem 2.1.4 (Ryjáček [19])

(i) Conjecture 1.1 and Conjecture 1.2 are equivalent.

(ii) Every 7-connected claw-free graph is hamiltonian.

It is well known that the line graph of the graph obtained by subdividing each edge of the Petersen graph exactly once is a 3-connected claw-free graph without a hamiltonian circuit. In this chapter, we consider the following problem: For 3-connected claw-free graphs, can high essential connectivity guarantee the existence of a hamiltonian circuit? This leads us to prove the following Theorem 2.1.5.

Theorem 2.1.5 Every 3-connected, essentially 11-connected line graph is hamiltonian.

Ryjáček [19] introduced the line graph closure of a claw-free graph and used it to show that a claw-free graph $G$ is hamiltonian if and only if its closure $\operatorname{cl}(G)$ is hamiltonian, where $c l(G)$ is a line graph. With this argument and using the fact that adding edges will not decrease the connectivity of a graph, The following corollary is obtained.

Corollary 2.1.6 Every 3-connected, essentially 11-connected claw-free graph is hamiltonian.

However, what is the smallest positive integer $k$ such that every 3-connected, essentially $k$-connected claw-free graph is hamiltonian? This question remains to be answered. Thus Corollary 2.1.6 below suggests that $4 \leq k \leq 11$. We fail to construct examples to show that there exists a 3-connected essentially 4-connected non-hamiltonian claw-free graph, and we conjecture that $k=4$.

\subsection{Reductions}

We shall introduce some of the reduction techniques to be used in the proof. 
Theorem 2.2.1 Let $G$ be a connected graph and let $G^{\prime}$ denote its reduction. Let $F(G)$ denote the minimum number of edges that must be added to $G$ so that the resulting graph has two edge-disjoint spanning trees. Each of the following holds.

(i) (Catlin [3]) If $H$ is a collapsible subgraph of $G$, then $G$ is collapsible if and only if $G / H$ is collapsible; $G$ is supereulerian if and only if $G / H$ is supereulerian.

(ii) (Catlin, Theorem 8 of [3]) If $G$ is reduced and if $|E(G)| \geq 3$, then $\delta(G) \leq 3$, and $2|V(G)|-|E(G)| \geq 4$.

(iii) (Catlin, Theorem 5 of [3]) A graph $G$ is reduced if and only if $G$ contains no nontrivial collapsible subgraphs. As circuits of length less than 4 are collapsible, a reduced graph does not have a circuit of length less than 4 .

Let $G$ be a connected, essentially 3-edge-connected graph such $L(G)$ is not a complete graph. The core of this graph $G$, denoted by $G_{0}$, is obtained by deleting all the vertices of degree 1 and contracting exactly one edge $x y$ or $y z$ for each path $x y z$ in $G$ with $d_{G}(y)=2$.

Lemma 2.2.2 (Shao [22]) Let $G$ be a connected, essentially 3-edge-connected graph $G$.

(i) $G_{0}$ is uniquely defined, and $\kappa^{\prime}\left(G_{0}\right) \geq 3$.

(ii) If $G_{0}$ is supereulerian, then $L(G)$ is hamiltonian.

A subgraph of $G$ isomorphic to a $K_{1,2}$ or a 2-circuit is called a 2-path or a $P_{2}$ subgraph of $G$. An edge cut $X$ of $G$ is a $P_{2}$-edge-cut of $G$ if at least two components of $G-X$ contain 2-paths. By the definition of a line graph, for a graph $G$, if $L(G)$ is not a complete graph, then $L(G)$ is essentially $k$-connected if and only if $G$ does not have a $P_{2}$ edge cut with size less than $k$. Since the core $G_{0}$ is obtained from $G$ by contractions (deleting a pendant edge is equivalent to contracting the same edge), every $P_{2}$-edge-cut of $G_{0}$ is also a $P_{2}$-edge-cut of $G$. Hence we have the following.

Lemma 2.2.3 Let $k>2$ be an integer, and let $G$ be a connected, essentially 3-edgeconnected graph. If $L(G)$ is essentially $k$-connected, then every $P_{2}$-edge-cut of $G_{0}$ has size at least $k$. 


\subsection{Proof of Theorem 2.1.5}

Throughout this section, we assume that $G$ is a graph such that $L(G)$ is 3-connected, essentially 11-connected, and that $L(G)$ is not a complete graph. Let $G_{0}$ denote the core of $G$ and $G_{0}^{\prime}$ denote the reduction of $G_{0}$. We shall show that $G_{0}^{\prime}=K_{1}$, and so $G_{0}$ is collapsible, which implies that $G_{0}$ is supereulerian. Hence by Lemma 2.2.2, $L(G)$ is hamiltonian.

By contradiction, we assume that $G_{0}^{\prime}$ is a nontrivial graph. By Theorem 2.2.1(iii),

$$
G_{0}^{\prime} \text { does not have a circuit of length less than } 4 \text {. }
$$

Since $L(G)$ is 3-connected, $G$ is essentially 3-edge-connected. By Lemma 2.2.2, $G_{0}^{\prime}$ is 3-edge-connected. By Theorem 2.2.1(ii), $D_{3}\left(G_{0}^{\prime}\right) \neq \emptyset$.

Lemma 2.3.1 For each $u, v, w \in V\left(G_{0}^{\prime}\right)$ such that $P=u v w$ is 2-path in $V\left(G_{0}^{\prime}\right)$, the edge cut $X=\left[\{u, v, w\}, V\left(G_{0}^{\prime}\right)-\{u, v, w\}\right]_{G_{0}^{\prime}}$ is a $P_{2}$-edge-cut of $G_{0}^{\prime}$ and $|X| \geq 11$.

Proof: Suppose that $G_{0}^{\prime}-X$ has components $H_{1}, H_{2}, \cdots, H_{c}$ with $c \geq 2$ and with $H_{1}=G_{0}^{\prime}[\{u, v, w\}]$ denoting a 2-path of $G_{0}^{\prime}$. To show that $X$ is a $P_{2}$-edge-cut of $G_{0}^{\prime}$, it suffices to show that for some $i \geq 2$,

$$
\left|V\left(H_{i}\right)\right| \geq 2 \text { and }\left|E\left(H_{i}\right)\right| \geq 2 \text {. }
$$

Suppose first that for some $i \geq 2,\left|E\left(H_{i}\right)\right|=1$. Since $H_{i}$ is a component of $G_{0}^{\prime}-X$, $\left|\left[\{u, v, w\}, V\left(H_{i}\right)\right]_{G_{0}^{\prime}}\right| \geq \kappa^{\prime}\left(G_{0}^{\prime}\right) \geq 3$, and so $G_{0}^{\prime}$ would have a circuit of length at most 3 , contrary to (2.1). Similarly, suppose that for some $i \geq 2$, we have $E\left(H_{i}\right)=\{x y\}$. Then by $\kappa^{\prime}\left(G_{0}^{\prime}\right) \geq 3$, each of $x$ and $y$ has degree at least 3 in $G_{0}^{\prime}$ and so $\left|\left[\{u, v, w\}, V\left(H_{i}\right)\right]_{G_{0}^{\prime}}\right| \geq 4$. It follows again that $G_{0}^{\prime}$ would have a circuit of length at most 3, contrary to (2.1). This proves $(2.2)$.

Thus $X$ is a $P_{2}$-edge-cut of $G_{0}^{\prime}$. Since $L(G)$ is essentially 11-connected, $|X| \geq 11$. 
Lemma 2.3.2 Every component of $G_{0}^{\prime}\left[D_{3}\left(G_{0}^{\prime}\right)\right]$ contains at most 2 vertices.

Proof: By contradiction, we assume that one component of $G_{0}^{\prime}\left[D_{3}\left(G_{0}^{\prime}\right)\right]$ contains at least 3 vertices, and so this component has three vertices $u, v, w$ such that $G_{0}^{\prime}[\{u, v, w\}]$ is connected. Thus $X=[\{u, v, w\}, V-\{u, v, w\}]$ is a $P_{2}$-edge-cut of $G_{0}^{\prime}$. Since $u, v, w \in$ $D_{3}\left(G_{0}^{\prime}\right),|X| \leq 5$, contrary to Lemma 2.3.1.

Define a real valued function

$$
f(x)=\frac{x-4}{x} \text {, over the interval }[3, \infty) .
$$

For each $v \in G_{0}^{\prime}$, define $l(v)=f\left(\operatorname{deg}_{G_{0}^{\prime}}(v)\right)$. Note that (i) of Lemma 2.3.3 below is a fact from Calculus and (ii) of Lemma 2.3.3 follows from (i) of Lemma 2.3.3.

Lemma 2.3.3 Each of the following holds.

(i) $f(x)$ is an increasing function.

(ii) If $\operatorname{deg}_{G_{0}^{\prime}}(v) \geq k$, then $l(v) \geq f(k)$.

Lemma 2.3.4 Suppose that $v \in D_{3}\left(G_{0}^{\prime}\right)$ is an isolated vertex of $G_{0}^{\prime}\left[D_{3}\left(G_{0}^{\prime}\right)\right]$ such that $v_{1}, v_{2}, v_{3}$ are the vertices adjacent to $v$ in $G_{0}^{\prime}$. Then $l\left(v_{1}\right)+l\left(v_{2}\right)+l\left(v_{3}\right) \geq 1$.

Proof: Since $v$ is an isolated vertex in $D_{3}\left(G_{0}^{\prime}\right), v_{i} \notin D_{3}\left(G_{0}^{\prime}\right)$. Relabelling the vertices if needed, we may assume that

$$
4 \leq \operatorname{deg}_{G_{0}^{\prime}}\left(v_{1}\right) \leq \operatorname{deg}_{G_{0}^{\prime}}\left(v_{2}\right) \leq \operatorname{deg}_{G_{0}^{\prime}}\left(v_{3}\right)
$$

For $i, j \in\{1,2,3\}$, by Lemma 2.3.1, $\operatorname{deg}_{G_{0}^{\prime}}\left(v_{i}\right)+\operatorname{deg}_{G_{0}^{\prime}}\left(v_{j}\right)-2+1=\mid\left[\left\{v, v_{i}, v_{j}\right\}, V\left(G_{0}^{\prime}\right)-\right.$ $\left.\left\{v, v_{i}, v_{j}\right\}\right] \mid \geq 11$, and so

$$
\operatorname{deg}_{G_{0}^{\prime}}\left(v_{i}\right)+\operatorname{deg}_{G_{0}^{\prime}}\left(v_{j}\right) \geq 12
$$

If $\operatorname{deg}_{G_{0}^{\prime}}\left(v_{1}\right) \geq 6$, then by (2.3) and by Lemma 2.3.3(ii), $l\left(v_{1}\right)+l\left(v_{2}\right)+l\left(v_{3}\right) \geq 3 f(6)=1$. Suppose then that $\operatorname{deg}_{G_{0}^{\prime}}\left(v_{1}\right)=5$. Then by (2.4), both $\operatorname{deg}_{G_{0}^{\prime}}\left(v_{2}\right) \geq 7$ and $\operatorname{deg}_{G_{0}^{\prime}}\left(v_{3}\right) \geq 7$. 
It follows by Lemma 2.3.3(ii) that $l\left(v_{1}\right)+l\left(v_{2}\right)+l\left(v_{3}\right) \geq f(5)+2 f(7) \geq 1$. Finally, we assume that $\operatorname{deg}_{G_{0}^{\prime}}\left(v_{1}\right)=4$. Then by $(4)$, both $\operatorname{deg}_{G_{0}^{\prime}}\left(v_{2}\right) \geq 8$ and $\operatorname{deg}_{G_{0}^{\prime}}\left(v_{3}\right) \geq 8$. It follows by Lemma 2.3.3(ii) that $l\left(v_{1}\right)+l\left(v_{2}\right)+l\left(v_{3}\right) \geq f(4)+2 f(8)=1$.

Lemma 2.3.5 Suppose that $v, w \in D_{3}\left(G_{0}^{\prime}\right)$ and $v w \in E\left(G_{0}^{\prime}\right)$. If $v_{1}, v_{2}, w$ are the vertices adjacent to $v$ in $G_{0}^{\prime}$ and if $v_{3}, v_{4}, v$ are the vertices adjacent to $w$ in $G_{0}^{\prime}$, then

(i) $v_{1}, v_{2}, v_{3}, v_{4}$ are mutually distinct vertices, and

(ii) both $l\left(v_{1}\right)+l\left(v_{2}\right) \geq 1$ and $l\left(v_{3}\right)+l\left(v_{4}\right) \geq 1$.

Proof: If $\left|\left\{v_{1}, v_{2}, v_{3}, v_{4}\right\}\right| \leq 4$, then $G_{0}^{\prime}$ could contain a circuit of length at most 3 , contrary to Theorem 2.2.1(iii). Thus Lemma 2.3.5(i) follows.

For $i \in\{1,2,3,4\}$, by Lemma 2.3.1, $\operatorname{deg}_{G_{0}^{\prime}}\left(v_{i}\right)-1+3=\left|\left[\left\{v, w, v_{i}\right\}, V-\left\{v, w, v_{i}\right\}\right]\right| \geq$ 11 , and so

$$
\operatorname{deg}_{G_{0}^{\prime}}\left(v_{i}\right) \geq 9 .
$$

It follows by (2.5) and Lemma 2.3.3(ii) that both $l\left(v_{1}\right)+l\left(v_{2}\right) \geq 2 f(9) \geq 1$ and $l\left(v_{3}\right)+$ $l\left(v_{4}\right) \geq 2 f(9) \geq 1$.

Let $d_{i}=\left|D_{i}\left(G_{0}^{\prime}\right)\right|$, for each $i \geq 3$. By Lemmas 2.3.2 Lemma 2.3.4 and Lemma 2.3.5, we have

$$
\begin{aligned}
d_{3} & =\sum_{v \in D_{3}} 1 \leq \sum_{v \in D_{3}} \sum_{u v \in E, u \notin D_{3}} l(u)=\sum_{u \notin D_{3}} \sum_{u v \in E, v \in D_{3}} l(u) \\
& =\sum_{i \geq 4} \sum_{u \in D_{i}} \sum_{u v \in E, v \in D_{3}} l(u) \leq \sum_{i \geq 4} \sum_{u \in D_{i}} i \cdot f(i) \\
& =\sum_{i \geq 4} \sum_{u \in D_{i}}(i-4)=\sum_{i \geq 4}(i-4) \cdot d_{i} .
\end{aligned}
$$

It follows by (2.6) that

$$
2(2|V(G)|-|E(G)|)=4|V(G)|-2|E(G)|=\sum_{i \geq 3}(4-i) \cdot d_{i}=d_{3}-\sum_{i \geq 4}(i-4) \cdot d_{i} \leq 0,
$$

contrary to Theorem 2.2.1. Thus $G_{0}^{\prime}=K_{1}$ and $G_{0}$ is supereulerian. By Lemma 2.2.2, $L(G)$ is hamiltonian. This completes the proof of Theorem 2.1.5. 


\section{Chapter 3}

\section{s-Hamiltonian and s-Hamiltonian Connected}

\subsection{The Problem and the Main Results}

Let $G$ be a graph. If $v \in V(G)$ and $H$ is a subgraph of $G$, then $N_{H}(v)$ denotes the set of vertices in $H$ that are adjacent to $v$ in $G$. Thus, $d_{H}(v)$, the degree of $v$ relative to $H$, is $\left|N_{H}(v)\right|$. We also write $d(v)$ for $d_{G}(v)$ and $N(v)$ for $N_{G}(v)$. If $C$ and $H$ are subgraphs of $G$, then $N_{C}(H)=\cup_{u \in V(H)} N_{C}(u)$, and $G-C$ denotes the subgraph of $G$ induced by $V(G)-V(C)$. Let $P=x_{1} x_{2} \cdots x_{m}$ denote a path of order $m$. To emphasize the end vertices of the path $P$, we also say that $P$ is an $\left(x_{1}, x_{m}\right)$-path. Define $N_{P}^{+}(u)=$ $\left\{x_{i+1} \in V(P): x_{i} \in N_{P}(u)\right\}$. So if $x_{m} \in N_{P}(u)$, then $\left|N_{P}^{+}(u)\right|=\left|N_{P}(u)\right|-1$. Two vertices are consecutive in $P$ if they are the ends of an edge in $E(P)$. Thus, each pair of vertices $x_{i}, x_{i+1}$ are consecutive in $P$ for any $i \in\{1, \cdots, m-1\}$. When $1 \leq i<j \leq m$, we use $\left[x_{i}, x_{j}\right]$ to denote the section $x_{i} x_{i+1} \cdots x_{j}$ of $P$ and $\left[x_{j}, x_{i}\right]$ to denote the section $x_{j} x_{j-1} \cdots x_{i}$ of $P$. If there is an $\left(x_{1}, x_{m}\right)$-path $P^{*}$ in $G$ such that $V(P) \subset V\left(P^{*}\right)$ and $\left|V\left(P^{*}\right)\right|>|V(P)|$, then we say that $P^{*}$ extends $P$. Let $C=x_{1} \cdots x_{m} x_{1}$ be a circuit. Define $N_{C}^{+}(H)=\left\{x_{i+1} \in V(C): x_{i} \in N_{C}(u)\right\}$, where the subscriptions are taken by modulo $m$. Two vertices are consecutive in $C$ if they are the ends of an edge in $E(C)$. If 
there is a circuit $C^{*}$ in $G$ such that $V(C) \subset V\left(C^{*}\right)$ and $\left|V\left(C^{*}\right)\right|>|V(C)|$, then we say that $C^{*}$ extends $C$.

The following sufficient conditions to ensure the existence of a Hamiltonian circuit in a simple graph $G$ of order $n \geq 3$ are well known.

Theorem 3.1.1 (Dirac [6]) If $\delta(G) \geq n / 2$, then $G$ is Hamiltonian.

Theorem 3.1.2 (Ore [17]) If $d(u)+d(v) \geq n$ for each pair of nonadjacent vertices $u, v \in V(G)$, then $G$ is Hamiltonian.

Theorem 3.1.3 (Fan [9]) If $G$ is a 2-connected graph and if $\max \{d(u), d(v)\} \geq n / 2$ for each pair of vertices $u, v \in V(G)$ with $d(u, v)=2$, then $G$ is Hamiltonian.

Theorem 3.1.4 (Chen [4]) If $G$ is a 2-connected graph and if $\max \{d(u), d(v)\} \geq n / 2$ for each pair of vertices $u, v \in V(G)$ with $1 \leq|N(u) \cap N(v)| \leq \alpha(G)-1$, then $G$ is Hamiltonian.

Theorem 3.1.5 (Chen et al [5]) If $G$ is a $k$-connected $(k \geq 2)$ graph and if $\max \{d(v)$ : $v \in I\} \geq n / 2$ for every independent set $I$ of order $k$ such that $I$ has two distinct vertices $x, y$ with $d(x, y)=2$, then $G$ is Hamiltonian.

Zhao et al recently proved Theorem 3.1.6 below, which unified and extended the above theorems.

Theorem 3.1.6 (Zhao et al [14]) If $G$ is a $k$-connected $(k \geq 2)$ graph of order $n$ and if $\max \{d(v): v \in I\} \geq n / 2$ for every independent set $I$ of order $k$ such that $I$ has two distinct vertices $x, y$ with $1 \leq|N(x) \cap N(y)| \leq \alpha(G)-1$, then $G$ is Hamiltonian.

In this chapter, we shall obtain sufficient conditions for $s$-Hamiltonian graphs and $s$-Hamiltonian connected graphs, respectively, as shown below. 
Theorem 3.1.7 Let $k, s$ be two integers with $k \geq s+2$ and $0 \leq s \leq n-3$. If $G$ is a $k$-connected graph of order $n$ and if $\max \{d(v): v \in I\} \geq(n+s) / 2$ for every independent set $I$ of order $k-s$ such that $I$ has two distinct vertices $x, y$ with $1 \leq|N(x) \cap N(y)| \leq$ $\alpha(G)+s-1$, then $G$ is s-Hamiltonian.

Theorem 3.1.8 Let $k, s$ be two integers with $k \geq s+3$ and $0 \leq s \leq n-2$. If $G$ is a $k$ connected graph of order $n$ and if $\max \{d(v): v \in I\} \geq(n+s+1) / 2$ for every independent set $I$ of order $k-s-1$ such that I has two distinct vertices $x, y$ with $1 \leq|N(x) \cap N(y)| \leq$ $\alpha(G)+s$, then $G$ is s-Hamiltonian connected.

Note that Theorem 3.1.6 is a special case of Theorem 3.1.7 when $s=0$. Applying Theorem 3.1.8 to the case when $s=0$, we get the following corollary.

Corollary 3.1.9 If $G$ is a $k$-connected $(k \geq 3)$ graph of order $n$ and if $\max \{d(v): v \in$ $I\} \geq(n+1) / 2$ for every independent set $I$ of order $k-1$ such that $I$ has two distinct vertices $x, y$ with $1 \leq|N(x) \cap N(y)| \leq \alpha(G)$, then $G$ is Hamiltonian-connected.

The following Lemma 3.1.10 is very important for the proof of the main theorems. A proof can also be found in [15].

Lemma 3.1.10 Let $G$ be a connected graph, $F=x_{1} \cdots x_{m}\left(x_{1}\right)$ be a longest path (or circuit) in $G$ and $H$ be a component of $G-V(F)$. If $x_{i}, x_{j} \in N_{F}(H)$ with $1 \leq i<j<m$, then

(i) $x_{i+1} x_{j+1} \notin E(G)$;

(ii) $N\left(x_{i+1}\right) \cap V(H)=\emptyset$;

(iii) $N_{F}^{+}(H) \cup\{x\}$ is an independent set of $G$, where $x \in V(H)$.

Theorem 3.1.7 and Theorem 3.1.8 will be proved in the following two sections, respectively. 


\subsection{Proof of Theorem 3.1.7}

Throughout this section, let $k, s$ denote two integers with $k \geq s+2$ and $0 \leq s \leq n-3$.

Lemma 3.2.1 [7] Let $G$ be a graph and $P=x_{1} \cdots x_{n}$ be a Hamiltonian-path of $G$. If $d\left(x_{1}\right)+d\left(x_{n}\right) \geq n$, then $G$ contains a Hamiltonian-circuit.

Lemma 3.2.2 Let $G$ be a $k$-connected graph of order $n, S \subseteq V(G)$ be a vertex set of order $s, C=x_{1} \cdots x_{m} x_{1}$ be a circuit of $G-S$ with $|V(C)|<n-s$ and $H$ be a component of $G-S-V(C)$. Then $G-S$ contains a circuit $C^{*}$ extending $C$, if one of the following holds:

(i) there exist two distinct vertices $x_{i}, x_{j} \in V(C)$ with $x_{i+1}, x_{j+1} \in N_{C}^{+}(H)$ such that $d\left(x_{i+1}\right) \geq(n+s) / 2$ and $d\left(x_{j+1}\right) \geq(n+s) / 2$, or

(ii) there exists a vertex $x_{i+1} \in N_{C}^{+}(H)$ and a vertex $y \in V(H)$ such that $d\left(x_{i+1}\right) \geq$ $(n+s) / 2$ and $d(y) \geq(n+s) / 2$.

Proof Since the proof when (ii) holds is similar to the proof when (i) holds, we only present the proof of the lemma assuming (i) holds. Let $x_{i}^{\prime}, x_{j}^{\prime} \in V(H)$ (possibly $x_{i}^{\prime}=x_{j}^{\prime}$ ) be such that $x_{i}^{\prime} x_{i}, x_{j}^{\prime} x_{j} \in E(G)$ and let $P$ be an $\left(x_{j}^{\prime}, x_{i}^{\prime}\right)$-path in $H$. Then $G[V(C \cup P)]$ has a Hamiltonian-path $P^{*}=\left[x_{i+1}, x_{j}\right] P\left[x_{i}, x_{1}\right]\left[x_{m}, x_{j+1}\right]$. Let $H^{\prime}=G-V(S \cup C \cup H)$. If $N_{H^{\prime}}\left(x_{i+1}\right) \cap N_{H^{\prime}}\left(x_{j+1}\right) \neq \emptyset$, let $z \in N_{H^{\prime}}\left(x_{i+1}\right) \cap N_{H^{\prime}}\left(x_{j+1}\right)$ and then $G-S$ has a circuit $C^{*}=$ $z\left[x_{i+1}, x_{j}\right] P\left[x_{i}, x_{1}\right]\left[x_{m}, x_{j+1}\right] z$ extending $C$. Now suppose that $N_{H^{\prime}}\left(x_{i+1}\right) \cap N_{H^{\prime}}\left(x_{j+1}\right)=\emptyset$ and so $d_{H^{\prime}}\left(x_{i+1}\right)+d_{H^{\prime}}\left(x_{j+1}\right) \leq\left|V\left(H^{\prime}\right)\right|$. If $N_{H-P}\left(x_{i+1}\right) \cup N_{H-P}\left(x_{j+1}\right) \neq \emptyset$, without loss of generality, let $y \in N_{H-P}\left(x_{i+1}\right) \cup N_{H-P}\left(x_{j+1}\right)$ and $y x_{i+1} \in E(G)$ and let $P^{\prime \prime}$ be an $\left(x_{i}^{\prime}, y\right)$-path in $H$. So $G-S$ has a circuit $C^{*}=x_{i} P^{\prime \prime}\left[x_{i+1}, x_{m}\right]\left[x_{1}, x_{i}\right]$ extending $C$. Now we can suppose that $N_{H-P}\left(x_{i+1}\right) \cup N_{H-P}\left(x_{j+1}\right)=\emptyset$ and so $d_{H-P}\left(x_{i+1}\right)+d_{H-P}\left(x_{j+1}\right)=0$. By (i) of Lemma 3.2.2, both $d\left(x_{i+1}\right) \geq(n+s) / 2$ and $d\left(x_{j+1}\right) \geq(n+s) / 2$. Thus,

$$
\begin{aligned}
d_{P^{*}}\left(x_{i+1}\right)+d_{P^{*}}\left(x_{j+1}\right)= & d\left(x_{i+1}\right)+d\left(x_{j+1}\right) \\
& -\left(d_{S \cup H^{\prime} \cup(H-P)}\left(x_{i+1}\right)+d_{S \cup H^{\prime} \cup(H-P)}\left(x_{j+1}\right)\right) \\
\geq & n+s-2 s-\left|V\left(H^{\prime}\right)\right| \geq\left|V\left(P^{*}\right)\right| .
\end{aligned}
$$

By Lemma 3.2.1, $G[V(C \cup P)]$ contains a Hamiltonian-circuit $C^{*}$ extending $C$. 
Lemma 3.2.3 Suppose that $G$ satisfies the hypothesis of Theorem 3.1.7. Let $S \subseteq V(G)$ be a vertex set with $|S|=s^{\prime} \leq s, C=x_{1} \cdots x_{m} x_{1}$ be a longest circuit of $G-S$ with $|V(C)|<n-s^{\prime}$ and $H$ be a component of $G-S-V(C)$. Then

(i) $\left|N_{C}(H)\right| \geq k-s$;

(ii) if $x \in V(H), x_{i} \in V(C)$ are such that $x x_{i} \in E(G)$, then $1 \leq\left|N(x) \cap N\left(x_{i+1}\right)\right| \leq$ $\alpha(G)+s-1$;

(iii) $d(x) \geq(n+s) / 2$ for each $x \in V(H)$ with $\left|N_{C}(x)\right| \geq 1$.

Proof (i) Since $C=x_{1} \cdots x_{m} x_{1}$ is a longest circuit of $G-S$ with $|V(C)|<n-s^{\prime}$, it follows that $H \neq \emptyset$ and $V(C)-N_{C}(H) \neq \emptyset$. By the facts that $N_{C}(H) \cup S$ separates $H$ and $G-H-\left(S \cup N_{C}(H)\right)$ and that $G$ is $k$-connected, we have $\left|N_{C}(H)\right|+|S| \geq k$ and so $\left|N_{C}(H)\right| \geq k-s^{\prime} \geq k-s$.

(ii) By Lemma 3.1.10 (iii), $N_{C}^{+}(H) \cup\{x\}$ is an independent set and so $\left|N_{C}(H)\right|=$ $\left|N_{C}^{+}(H)\right| \leq \alpha(G)-1$. It follows that $1 \leq\left|N(x) \cap N\left(x_{i+1}\right)\right| \leq\left|N_{C}(H) \cup S\right| \leq \alpha(G)+s^{\prime}-1 \leq$ $\alpha(G)+s-1$.

(iii) Suppose, to the contrary, that there exists an $x \in V(H)$ with $\left|N_{C}(x)\right| \geq 1$ and with $d(x)<(n+s) / 2$. Let $x_{i} \in N_{C}(x)$. By Lemma 3.1.10 (iii) and by the fact that $\left|N_{C}^{+}(H)\right|=\left|N_{C}(H)\right| \geq k-s, G$ has an independent set $J=J^{\prime} \cup\{x\}$ of order $k-s$ with $x_{i+1} \in J^{\prime} \subseteq N_{C}^{+}(H)$. By (ii), $1 \leq\left|N(x) \cap N\left(x_{i+1}\right)\right| \leq \alpha(G)+s-1$. Hence by the hypothesis of Theorem 3.1.7 and by the fact that $d(x)<(n+s) / 2$, there must exist an $x_{l+1} \in J^{\prime}$ satisfying $d\left(x_{l+1}\right) \geq(n+s) / 2$. By $(\mathrm{i}),\left|N_{C}^{+}(H)\right|=\left|N_{C}(H)\right| \geq k-s \geq 2$, and so there exists an $x_{j+1} \in N_{C}^{+}(H)-\left\{x_{l+1}\right\}$. Since $x_{j+1} \in N_{C}^{+}(H), x_{j} \in N_{C}(H)$ and we may assume $y \in V(H)$ with $y x_{j} \in E(G)$ (possible $y=x$ ). By (ii), we have $1 \leq\left|N(y) \cap N\left(x_{j+1}\right)\right| \leq \alpha(G)+s-1$. Similarly, $G$ has an independent set $J_{1}=J_{1}^{\prime} \cup\{y\}$ of order $k-s$, where $x_{j+1} \in J_{1}^{\prime} \subseteq N_{C}^{+}(H)-\left\{x_{l+1}\right\}$. By the hypothesis of Theorem 3.1.7, there exists a $z \in J_{1}$ such that $d(z) \geq(n+s) / 2$. Consequently, either $z \in N_{C}^{+}(H)$, whence by Lemma 3.2.2 (i), $G-S$ has a circuit $C^{*}$ extending $C$; or $z=y$, whence by Lemma 3.2.2 (ii), $G-S$ has a circuit $C^{*}$ extending $C$. In either case, a contradiction to the assumption that $C$ is a longest circuit of $G-S$ is obtained.

Proof of Theorem 3.1.7 Let $G$ be a graph satisfying the hypothesis of Theorem 3.1.7. Suppose, to the contrary, that $G$ is not $s$-Hamiltonian. Then there exists a vertex set 
$S \subseteq V(G)$ with $|S|=s^{\prime} \leq s$ such that $G-S$ does not have a Hamiltonian-circuit. By the fact that $k-s^{\prime} \geq k-s \geq 2, G-S$ is 2 -connected. We may assume that

$$
C=x_{1} \cdots x_{m} x_{1} \text { is a longest circuit in } G-S \text {. }
$$

Then $|V(C)|<n-s^{\prime}$. Let $H$ be a component of $G-S-V(C)$. By Lemma 3.2.3 (i), we have $\left|N_{C}(H)\right| \geq k-s \geq 2$. Choose $x_{i}, x_{j} \in N_{C}(H)$ to be such that

$$
X \cap N_{C}(H)=\emptyset, \text { and }|X| \text { is minimum, }
$$

where $X=\left\{x_{i+1}, \cdots, x_{j-1}\right\}$. Then $|X|>0$. Otherwise, there exist $y_{i}, y_{i+1} \in V(H)$ such that $x_{i} y_{i} \in E(G), x_{i+1} y_{i+1} \in E(G)$ ( $y_{i}$ and $y_{i+1}$ might be the same vertex). Let $P_{H}\left[y_{i}, y_{i+1}\right]$ be a $\left(y_{i}, y_{i+1}\right)$-path in $H$. Then $C^{*}=\left[x_{1}, x_{i}\right] P_{H}\left[y_{i}, y_{i}+1\right]\left[x_{i+1}, x_{m}\right] x_{1}$ is a circuit extending $C$, contrary to (3.1). By Lemma 3.2.3 (iii), for each vertex $x \in V(H)$ with $\left|N_{C}(x)\right| \geq 1, d(x) \geq(n+s) / 2$. Since $N(x) \cup\{x\} \subseteq V(H) \cup N_{C}(H) \cup S$ for each $x \in V(H),|V(H)|+\left|N_{C}(H)\right|+|S| \geq(n+s) / 2+1$, and then

$$
|V(H)|+\left|N_{C}(H)\right| \geq \frac{n-s^{\prime}}{2}+1 .
$$

Claim 1. $G-S-V(C)$ has only one component $H=G-S-V(C)$ and $|X|<|V(H)|$.

Proof. Suppose, to the contrary, that $G-S-V(C)$ has at least two components. Assume that $H$ is the component with the smallest order and let $H^{*}=G-S-V(C \cup H)$. Since $|V(H)|$ is minimized, $|V(H)| \leq\left|V\left(H^{*}\right)\right|$. It follows by (3.3) and $\left|N_{C}(H)\right| \geq 2$ that

$$
\begin{aligned}
|X| & \leq \frac{|V(C)|-\left|N_{C}(H)\right|}{\left|N_{C}(H)\right|}=\frac{n-\left|V\left(H^{*}\right)\right|-s^{\prime}-\left(|V(H)|+\left|N_{C}(H)\right|\right)}{\left|N_{C}(H)\right|} \\
& \leq \frac{\left(n-s^{\prime}\right) / 2-1-\left|V\left(H^{*}\right)\right|}{\left|N_{C}(H)\right|} \leq \frac{|V(H)|+\left|N_{C}(H)\right|-2-\left|V\left(H^{*}\right)\right|}{\left|N_{C}(H)\right|} \\
& =\frac{|V(H)|-\left|V\left(H^{*}\right)\right|}{\left|N_{C}(H)\right|}+\frac{\left|N_{C}(H)\right|-2}{\left|N_{C}(H)\right|} .
\end{aligned}
$$

Then as $|V(H)| \leq\left|V\left(H^{*}\right)\right|$, (4) implies $|X|<1$, contrary to the fact that $|X|>0$. Hence, $H$ is the only component of $G-S-V(C)$. Since $\left|N_{C}(H)\right| \geq 2$, we have that $|X|<|V(H)|$. 
Choose $x_{i}^{\prime}, x_{j}^{\prime} \in V(H)$ with $x_{i} x_{i}^{\prime} \in E(G), x_{j} x_{j}^{\prime} \in E(G)$ to be such that $\left|V\left(P^{\prime}\right)\right|$ is as large as possible, where $P^{\prime}$ is an $\left(x_{i}^{\prime}, x_{j}^{\prime}\right)$-path in $H$. Then $C^{\prime}=\left[x_{1}, x_{i}\right] P^{\prime}\left[x_{j}, x_{m}\right] x_{1}$ is a circuit such that

$$
V(C) \backslash X \subseteq V\left(C^{\prime}\right) \text { and }\left|V\left(C^{\prime}\right)\right| \text { is maximized. }
$$

By (3.5), $C^{\prime}$ is a longest path containing $V(C) \backslash X$ and so by applying Lemma 3.2.3 and the argument on $C$ to $C^{\prime}$, it follows that $G-S-V\left(C^{\prime}\right)$ has only one component $H^{\prime}$ and that $H^{\prime}=G\left[X \cup V\left(H-P^{\prime}\right)\right]$. By (3.2) and the fact that $|X|>0, H-P^{\prime}=\emptyset$. Otherwise, $H^{\prime}$ is connected while $G\left[X \cup\left(H-P^{\prime}\right)\right]$ is disconnected, a contradiction. Therefore $P^{\prime}$ is a path of order $|V(H)|$. By the fact that $|X|<|V(H)|$, we have $\left|V\left(C^{\prime}\right)\right|=|V(C)|-|X|+|V(H)|>$ $|V(C)|$, contrary to (3.1). This completes the proof of Theorem 3.1.7.

\subsection{Proof of Theorem 3.1.8}

Lemma 3.3.1 Let $G$ be a graph and $P=x_{1} \cdots x_{n}$ be a Hamiltonian-path of $G$. If $d\left(x_{1}\right)+d\left(x_{n}\right) \geq n+1$, then for any edge $e=x_{i} x_{i+1} \in E(P), G$ has a Hamiltonian-circuit $C$ such that $e \in(C)$.

Proof Let $T=\left\{x_{j} \mid x_{1} x_{j+1} \in E, x_{j+1} \in V(P)\right\}$. Then

$$
\left|T \cap N\left(x_{n}\right)\right|=|T|+\left|N\left(x_{n}\right)\right|-\left|T \cup N\left(x_{n}\right)\right| \geq n+1-(n-1)=2 .
$$

That means there exists $x_{j} \in T \cap N\left(x_{n}\right)-\left\{x_{i}\right\}$, and so $G$ has a Hamiltonian-circuit $C=\left[x_{1}, x_{j}\right]\left[x_{n}, x_{j+1}\right] x_{1}$. Clearly, $E(P)-\left\{x_{j} x_{j+1}\right\} \subseteq E(C)$, and so $e=x_{i} x_{i+1} \in E(C)$. Thus the lemma holds.

Lemma 3.3.2 Let $G$ be a k-connected graph of order $n, S \subseteq V(G)$ be a vertex set with $|S|=s^{\prime} \leq s, P=x_{1} \cdots x_{m}$ be a path of $G-S$ with $|V(P)|<n-s$ and $H$ be a component of $G-S-V(P)$. Then $G-S$ contains a path $P^{*}$ extending $P$, if one of the following holds:

(i) there exist two distinct vertices $x_{i}, x_{j} \in V(P)$ with $x_{i+1}, x_{j+1}$ in $N_{P}^{+}(H)$ such that $d\left(x_{i+1}\right) \geq(n+s+1) / 2$ and $d\left(x_{j+1}\right) \geq(n+s+1) / 2$, or 
(ii) there exists a vertex $x_{i+1} \in N_{P}^{+}(H)$ and a vertex $y \in V(H)$ such that $d\left(x_{i+1}\right) \geq$ $(n+s+1) / 2$ and $d(y) \geq(n+s+1) / 2$.

Proof Since the proof when (ii) holds is similar to the proof when (i) holds, we shall only present the proof of the Lemma 3.3 .2 assuming (i) holds. Let $x_{i}^{\prime}, x_{j}^{\prime} \in V(H)$ with $x_{i}^{\prime} x_{i}, x_{j}^{\prime} x_{j} \in E(G)$ and let $P^{\prime}$ be an $\left(x_{j}^{\prime}, x_{i}^{\prime}\right)$-path in $H$. Define $G_{1}$ to be the graph obtained from $G$ by adding a new edge $x_{1} x_{m}$ if $x_{1} x_{m} \notin E(G)$ and to be $G$ if $x_{1} x_{m} \in E(G)$. Then we have an $\left(x_{i+1}, x_{j+1}\right)$-path $P_{1}=\left[x_{i+1}, x_{j}\right] P^{\prime}\left[x_{i}, x_{1}\right]\left[x_{m}, x_{j+1}\right]$ with $V\left(P_{1}\right)=V(P) \cup$ $V\left(P^{\prime}\right)$ in $G_{1}$. Moreover, $x_{1} x_{m}$ is an edge of $P_{1}$. Let $H^{*}=G-V(S \cup P \cup H)$. If $N_{H^{*}}\left(x_{i+1}\right) \cap N_{H^{*}}\left(x_{j+1}\right) \neq \emptyset$, let $z \in N_{H^{*}}\left(x_{i+1}\right) \cap N_{H^{*}}\left(x_{j+1}\right)$ and then $G\left[V\left(P_{1}\right) \cup\{z\}\right]$ has a Hamiltonian-circuit $C$ such that $x_{1} x_{m} \in E(C)$. Therefore, $C-\left\{x_{1} x_{m}\right\}$ is an $\left(x_{1}, x_{m}\right)$ path in $G-S$ which extends $P$. Now suppose that $N_{H^{*}}\left(x_{i+1}\right) \cap N_{H^{*}}\left(x_{j+1}\right)=\emptyset$ and so we have $d_{H^{*}}\left(x_{i+1}\right)+d_{H^{*}}\left(x_{j+1}\right) \leq\left|V\left(H^{*}\right)\right|$. If $N_{H-P^{\prime}}\left(x_{i+1}\right) \cup N_{H-P^{\prime}}\left(x_{j+1}\right) \neq \emptyset$, without loss of generality, let $y \in N_{H-P^{\prime}}\left(x_{i+1}\right) \cup N_{H-P^{\prime}}\left(x_{j+1}\right)$ and $y x_{i+1} \in E(G)$ and let $P^{\prime \prime}$ be an $\left(x_{i}^{\prime}, y\right)$-path in $H$. So $G-S$ has a path $P^{*}=\left[x_{1}, x_{i}\right] P^{\prime \prime}\left[x_{i+1}, x_{m}\right]$ extending $P$. Now we can suppose that $N_{H-P^{\prime}}\left(x_{i+1}\right) \cup N_{H-P^{\prime}}\left(x_{j+1}\right)=\emptyset$ and so $d_{H-P^{\prime}}\left(x_{i+1}\right)+d_{H-P^{\prime}}\left(x_{j+1}\right)=0$. Since $d\left(x_{i+1}\right) \geq(n+s+1) / 2$ and $d\left(x_{j+1}\right) \geq(n+s+1) / 2$, we have

$$
\begin{aligned}
d_{P_{1}}\left(x_{i+1}\right)+d_{P_{1}}\left(x_{j+1}\right)= & d\left(x_{i+1}\right)+d\left(x_{j+1}\right) \\
& -\left(d_{S \cup H^{*} \cup\left(H-P^{\prime}\right)}\left(x_{i+1}\right)+d_{S \cup H^{*} \cup\left(H-P^{\prime}\right)}\left(x_{j+1}\right)\right) \\
\geq & n+s+1-2 s-\left|V\left(H^{*}\right)\right| \geq\left|V\left(P_{1}\right)\right|+1 .
\end{aligned}
$$

By Lemma 3.3.1, $G_{1}\left[V\left(P_{1}\right)\right]$ contains a Hamiltonian-circuit $C$ such that $x_{1} x_{m} \in E(C)$, and then $C-\left\{x_{1} x_{m}\right\}$ is an $\left(x_{1}, x_{m}\right)$-path $P^{*}$ in $G-S$ extending $P$.

By a proof similar to that for Lemma 3.2.3, we obtain the following lemma.

Lemma 3.3.3 Suppose that $G$ satisfies the hypothesis of Theorem 3.1.8. Let $S \subseteq V(G)$ be a vertex set with $|S|=s^{\prime} \leq s, P=x_{1} \cdots x_{m}$ be a longest path of $G-S$ with $|V(P)|<n-s^{\prime}$ and $H$ be a component of $G-S-V(P)$. Then

(i) $\left|N_{P}(H)\right| \geq k-s$;

(ii) if $x \in V(H), x_{i} \in V(P)$ with $x x_{i} \in E$, then $1 \leq\left|N(x) \cap N\left(x_{i+1}\right)\right| \leq \alpha(G)+s$;

(iii) $d(x) \geq(n+s+1) / 2$ for each $x \in V(H)$ with $\left|N_{P}(x)\right| \geq 1$. 
Proof of Theorem 3.1.8 Let $G$ be a graph satisfying the hypothesis of Theorem 3.1.8. Suppose, to the contrary, that $G-S$ is not Hamiltonian-connected for some vertex set $S \subseteq V(G)$ with $|S|=s^{\prime} \leq s$. Then there exists a pair of vertices, say $x$ and $y$, such that $G-S$ does not have a Hamiltonian $(x, y)$-path. Since $k-s^{\prime} \geq k-s \geq 3, G-S$ is 3-connected and we can choose

$$
P=x_{1} x_{2} \cdots x_{m} \text { to be a longest }(x, y) \text {-path in } G-S,
$$

where $x=x_{1}, y=x_{m}$. Then $|V(P)|<n-s^{\prime}$. Let $H$ be a component of $G-S-V(P)$. By Lemma 3.3.3 (i), we have $\left|N_{P}(H)\right| \geq k-s \geq 3$. Choose $x_{i}, x_{j} \in N_{P}(H)$ to be such that

$$
X \cap N_{P}(H)=\emptyset \text { and }|X| \text { is minimum, }
$$

where $X=\left\{x_{i+1}, \cdots, x_{j-1}\right\}$. Then $|X|>0$. Otherwise, there exist $y_{i}, y_{i+1} \in V(H)$ such that $x_{i} y_{i} \in E(G), x_{i+1} y_{i+1} \in E(G)$ ( $y_{i}$ and $y_{i+1}$ might be the same vertex). Let $P_{H}\left[y_{i}, y_{i}+1\right]$ be a $\left(y_{i}, y_{i+1}\right)$-path in $H$. Then $P^{*}=\left[x_{1}, x_{i}\right] P_{H}\left[y_{i}, y_{i+1}\right]\left[x_{i+1}, x_{m}\right]$ is an $\left(x_{1}, x_{m}\right)$-path extending $P$, contrary to (3.6). By Lemma 3.3.3 (iii), for each vertex $x \in$ $V(H)$ with $\left|N_{C}(x)\right| \geq 1, d(x) \geq(n+s+1) / 2$. Since for each $x \in V(H), N(x) \cup\{x\} \subseteq$ $V(H) \cup N_{P}(H) \cup S$,

$$
|V(H)|+\left|N_{P}(H)\right| \geq\left(n-s^{\prime}\right) / 2+3 / 2 .
$$

By a proof similar to that for the Claim 1 in the proof of Theorem 1.7, we get the following.

Claim 2. $G-S-V(P)$ has only one component $H=G-S-V(P)$ and $|X|<|V(H)|$.

Choose $x_{i}^{\prime}, x_{j}^{\prime} \in V(H)$ with $x_{i}^{\prime}, x_{j}^{\prime} \in V(H)$ to be such that $\left|V\left(P^{\prime}\right)\right|$ is as large as possible, where $P^{\prime}$ is an $\left(x_{i}^{\prime}, x_{j}^{\prime}\right)$-path in $H$. Then $P^{*}=\left[x_{1}, x_{i}\right] P^{\prime}\left[x_{j}, x_{m}\right]$ is a path such that

$$
V(P) \backslash X \subseteq V\left(P^{*}\right) \text { and }\left|V\left(P^{*}\right)\right| \text { is maximized. }
$$

By (3.9), $P^{*}$ is a longest path containing $V(P) \backslash X$ and so by applying Lemma 3.3.3 and the argument on $P$ to $P^{*}$, it follows that $G-S-V\left(P^{*}\right)$ has only one component $H^{\prime}$ and that $H^{\prime}=G\left[X \cup V\left(H-P^{\prime}\right)\right]$. By (3.7) and the fact that $|X|>0, H-P^{\prime}=\emptyset$. Otherwise, $H^{\prime}$ is connected while $X \cup\left(H-P^{\prime}\right)$ is disconnected, a contradiction. Therefore, $P^{\prime}$ is a path of order $|V(H)|$. By the fact that $|X|<|V(H)|$, we have $\left|V\left(P^{*}\right)\right|=|V(P)|-|X|+|V(H)|>$ $|V(P)|$, contrary to (3.6). This completes the proof of Theorem 3.1.8. 


\section{Chapter 4}

\section{Degree Sequence and Supereulerian Graphs}

\subsection{The Problem and the Main Results}

Let $G$ be a graph with vertex set $V(G)$ and edge set $E(G)$. A vertex $v \in V(G)$ is called a pendent vertex if $d(v)=1$ and denote the set of all pendent vertices of $G$ by $D_{1}(G)$. An edge $e \in E(G)$ is called a pendent edge if one of its endpoints is a pendent vertex. If $v \in V(G)$, then $N(v)=\{u: u v \in E(G)\}$. If $T \subseteq V(G)$, then $N(T)=\{u \in V(G) \backslash T$ : $u v \in E(G)$ and $v \in T\}$.

In [26], Zhang et al. proved the following theorem.

Theorem 4.1.1 [26] Every bipartite graphic sequence with the minimum degree $\delta \geq 2$ has a realization that admits a nowhere-zero 4-flow.

In this paper, we first get the following result.

Theorem 4.1.2 If $d=\left(d_{1}, d_{2}, \cdots, d_{n}\right) \in \mathcal{G}$ is a nonincreasing sequences with $d_{n} \geq 2$, then $d$ has a supereulerian realization. 
In [12], Jaeger proved the following result.

Theorem 4.1.3 [12] Every supereulerian graph admits a nowhere-zero 4-flow.

Combining Theorem 4.1.3, we get a result analogous to Theorem 4.1.1.

Theorem 4.1.4 If $d=\left(d_{1}, d_{2}, \cdots, d_{n}\right) \in \mathcal{G}$ is a nonincreasing sequences with $d_{n} \geq 2$, then $d$ has a realization that admits a nowhere-zero 4-flow.

Furthermore, we get a result about line-hamiltonian sequence as follows.

Theorem 4.1.5 If $d=\left(d_{1}, d_{2}, \cdots, d_{n}\right)$ is a nonincreasing graphic sequence with $n \geq 3$, then the following are equivalent.

(i) d is line-hamiltonian.

(ii) $d \in \mathcal{G}$ and either $d_{1}=n-1$, or

$$
\sum_{d_{i}=1} d_{i} \leq \sum_{d_{j} \geq 2}\left(d_{j}-2\right)
$$

(iii) d has a realization $G$ such that $G-D_{1}(G)$ is supereulerian.

\subsection{Collapsible Sequences}

Theorem 4.2.1 Let $G$ be a connected graph. Each of the following holds.

(i) (Catlin, Corollary of Lemma 3, [3]) If $H$ is a collapsible subgraph of $G$, then $G$ is collapsible if and only if $G / H$ is collapsible.

(ii) (Catlin, Corollary 1, [3]) If $G$ contains a spanning tree $T$ such that each edge of $T$ is contained in a collapsible subgraph of $G$, then $G$ is collapsible.

(iii) (Caltin, Theorem \%, [3]) $C_{2}, K_{3}$ are collapsible.

(iv) (Caltin, Theorem 2, [3]) If $G$ is collapsible, then $G$ is supereulerian. 
Theorem 4.2.1(ii) and (iii) imply Corollary 4.2.2 (i); Theorem 4.2.1(i) and (iii) imply Corollary 4.2.2(ii).

Corollary 4.2.2 (i) If every edge of a spanning tree of $G$ lies in a $K_{3}$, then $G$ is collapsible.

(ii) If $G-v$ is collapsible and if $v$ has degree at least 2 in $G$, then $G$ is collapsible.

Corollary 4.2.3 If $d=\left(d_{1}, d_{2}, \cdots, d_{n}\right)$ is a nonincreasing graphic sequence with $d_{1}=$ $n-1$ and $d_{n} \geq 2$, then every realization of $d$ is collapsible.

Proof. Let $G$ be a realization of $d$ with $N\left(v_{1}\right)=\left\{v_{2}, \cdots, v_{n}\right\}$ and let $T$ be the spanning tree with $E(T)=\left\{v_{1} v_{i}: 2 \leq i \leq n\right\}$. Since $d_{n} \geq 2$ and $N\left(v_{1}\right)=\left\{v_{2}, \cdots, v_{n}\right\}$, for any $v_{i} \in\left\{v_{1} v_{i}: 2 \leq i \leq n\right\}$, there is $v_{j} \in\left\{v_{1} v_{i}: 2 \leq i \leq n\right\} \backslash\left\{v_{i}\right\}$ such that $v_{i} v_{j} \in E(G)$. So every edge of $T$ lies in a $K_{3}$, and by Theorem 4.2.1(ii), $G$ is collapsible.

Lemma 4.2.4 If $d=\left(d_{1}, d_{2}, \cdots, d_{n}\right)$ is a nonincreasing graphic sequence with $d_{3}=\cdots=$ $d_{n}=3$, then $d$ is collapsible.

Proof. Let $v_{1}, v_{2}$ be two vertices and let

$$
S=\left\{\begin{aligned}
\left\{s_{1}, s_{2}, \cdots, s_{d_{2}}\right\} & : \text { if } d_{2} \text { is even } \\
\left\{s_{1}, s_{2}, \cdots, s_{d_{2}-1}\right\} & : \text { if } d_{2} \text { is odd }
\end{aligned}\right.
$$

be a set of vertices other than $\left\{v_{1}, v_{2}\right\}$ and let $T=\left\{t_{1}, t_{2}, \cdots, t_{d_{1}-d_{2}}\right\}$ be a set of $d_{1}-d_{2}$ vertices other than $S \cup\left\{v_{1}, v_{2}\right\}$. Let $H$ denote the graph obtained from $\left\{v_{1}, v_{2}\right\} \cup S \cup T$ by joining $v_{2}$ to each vertex of $S$ and joining $v_{1}$ to each vertex of $S \cup T$ (if $d_{2}$ is odd, then we also join $v_{1}$ and $\left.v_{2}\right)$. Note that $d_{H}\left(v_{1}\right)=d_{2}+d_{1}-d_{2}=d_{1}, d_{H}\left(v_{2}\right)=d_{2}, d_{H}(s)=2$ for $s \in S$ and $d_{H}(t)=1$ for $t \in T$.

Let $C=t_{1} t_{2} \cdots t_{d_{2}-d_{1}} t_{1}$ be a cycle passing all vertices of $T$ and let $H^{\prime}=H \cup E(C)$. As $|S|$ is even, we join all vertices of $S$ in pairs (i.e., $\left.s_{1} s_{2}, s_{3} s_{4}, \cdots\right)$ in $H^{\prime}$ and denote 
the resulting graph by $H^{\prime \prime}$. Note that $d_{H^{\prime \prime}}\left(v_{1}\right)=d_{1}, d_{H^{\prime \prime}}\left(v_{2}\right)=d_{2}$ and $d_{H^{\prime \prime}}(v)=3$ for $v \in S \cup T$.

Also note that

$$
\left|V\left(H^{\prime \prime}\right)\right|= \begin{cases}2+d_{1} & : \text { if } d_{2} \text { is even } \\ 1+d_{1} & : \text { if } d_{2} \text { is odd }\end{cases}
$$

Let $m=n-\left|V\left(H^{\prime \prime}\right)\right|$ and so

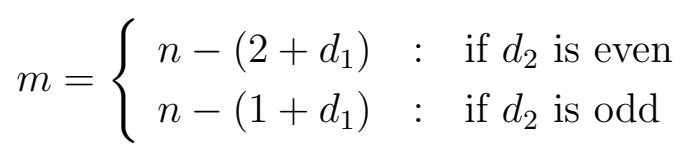

is even. By the construction of $H^{\prime \prime}, H^{\prime \prime}$ contains a triangle $v_{1} s_{1} s_{2}$. We subdivide $v_{1} s_{1}$ and $v_{1} s_{2} \frac{m}{2}$ times, respectively, and let $x_{1}, x_{2}, \cdots, x_{\frac{m}{2}}$ and $y_{1}, y_{2}, \cdots, y_{\frac{m}{2}}$ be the subdivision vertices of $v_{1} s_{1}$ and $v_{1} s_{2}$, respectively. Then for $1 \leq j \leq \frac{m}{2}$, we join $x_{j} y_{j}$ and denote the resulting graph by $G$ (see Figure 1 ). Hence, by the construction of $G, G$ is a realization of $d$.

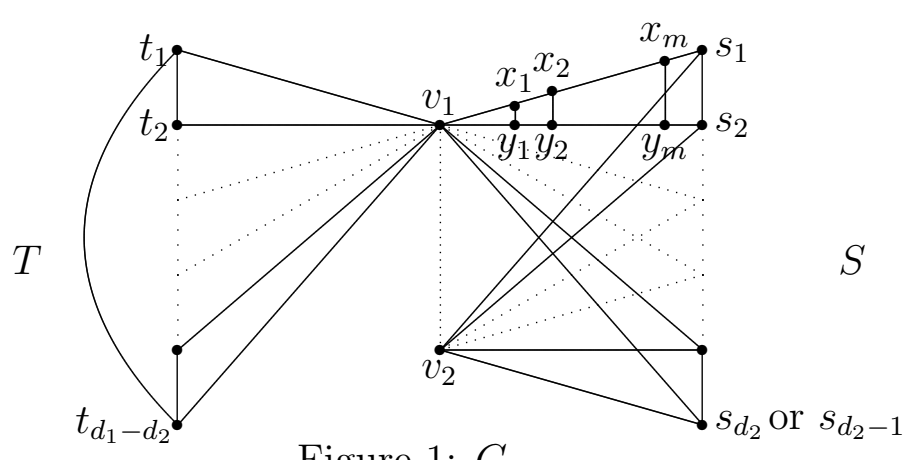

Figure 1: $G$

By Theorem 4.2.1(iii), $K_{3}$ is collapsible. If we contract $v_{1} x_{1} y_{1}$, then we get a triangle $v_{1} x_{2} y_{2}$ and if we contract $v_{1} x_{2} y_{2}$, then we get a triangle $v_{1} x_{3} y_{3}$ and so on until we get $v_{1} s_{1} s_{2}$. After contracting $v_{1} t_{1} t_{2}$ we get a graph in which each edge lies in a triangle. By Theorem 4.2.2(i), $G$ is collapsible.

Theorem 4.2.5 (Ex. 1.5.7(a) on page 11, [2]) Let $d=\left(d_{1}, d_{2}, \cdots, d_{n}\right)$ be a nonincreasing sequence. Then $d$ is graphic if and only if $d^{\prime}=\left(d_{2}-1, d_{3}-1, \cdots, d_{d_{1}+1}-1, d_{d_{1}+2}, \cdots, d_{n}\right)$ is graphic. 
Lemma 4.2.6 If $d=\left(d_{1}, d_{2}, \cdots, d_{n}\right)$ is a nonincreasing sequence with $n \geq 4$ and $d_{n}=3$, then $d$ is graphic if and only if $d^{\prime}=\left(d_{1}-1, d_{2}-1, d_{3}-1, d_{4}, \cdots, d_{n-1}\right)$ is graphic.

Proof. Let $G$ be a realization of $d$ with $d\left(v_{i}\right)=d_{i}$ for $1 \leq i \leq n$. If $N\left(v_{n}\right)=\left\{v_{1}, v_{2}, v_{3}\right\}$, then $G-v_{n}$ is a realization of $d^{\prime}$. So it is suffices to prove the following claim.

Claim 1 There is a realization $G$ with $d\left(v_{i}\right)=d_{i}$ for $1 \leq i \leq n$ and $N\left(v_{n}\right)=\left\{v_{1}, v_{2}, v_{3}\right\}$.

Proof. Choose $G$ to be a realization of $d$ such that $\left|N\left(v_{n}\right) \cap\left\{v_{1}, v_{2}, v_{3}\right\}\right|$ is as large as possible. If $\left|N\left(v_{n}\right) \cap\left\{v_{1}, v_{2}, v_{3}\right\}\right|=3$, then we are done. Suppose that $\mid N\left(v_{n}\right) \cap$ $\left\{v_{1}, v_{2}, v_{3}\right\} \mid<3$. Then $v_{n} v_{i} \notin E(G)$ for some $i \in\{1,2,3\}$. As $d\left(v_{n}\right)=3$, there exists $x \in$ $N\left(v_{n}\right)$ such that $x \notin\left\{v_{1}, v_{2}, v_{3}\right\}$. Then there must exist $v_{i}^{\prime} \in N\left(v_{i}\right)$ such that $v_{i}^{\prime} x \notin E(G)$, otherwise $|N(x)| \geq\left|N\left(v_{i}\right) \cup\left\{v_{n}\right\}\right|=d_{i}+1$, contrary to the fact that $d(x) \leq d_{3} \leq d_{i}$. Let $G^{\prime}=G-\left\{v_{i} v_{i}^{\prime}, v_{n} x\right\}+\left\{v_{i} v_{n}, v_{i}^{\prime} x\right\}$. Then $\left|N_{G^{\prime}}\left(v_{n}\right) \cap\left\{v_{1}, v_{2}, v_{3}\right\}\right|>\left|N_{G}\left(v_{n}\right) \cap\left\{v_{1}, v_{2}, v_{3}\right\}\right|$, contradicting the choice of $G$.

Conversely, if $G^{\prime}$ is a realization of $d^{\prime}$, then can get a realization $G$ of $d$ by adding a new vertex $u$ to $G^{\prime}$ and joining $u$ to the vertices of degree $d_{1}-1, d_{2}-1, d_{3}-1$ in $G^{\prime}$, respectively.

Theorem 4.2.7 If $d=\left(d_{1}, d_{2}, \cdots, d_{n}\right)$ is a nonincreasing graphic sequence with $n \geq 4$ and $d_{n} \geq 3$, then $d$ has a collapsible realization.

Proof. We argue by induction on $n$. First we assume that $n=4$. Then the realization of $d$ must be a $K_{4}$. By Theorem 4.2.1, $K_{4}$ is collapsible.

Next we assume that $n \geq 5$. If $d_{n} \geq 4$, then $d_{2}-1 \geq d_{3}-1 \geq \cdots \geq d_{d_{1}+1}-1 \geq 3$ and $d_{d_{1}+2} \geq \cdots \geq d_{n} \geq 3$. By Theorem 4.2 .5 and the induction hypothesis, $\left(d_{2}-\right.$ $\left.1, d_{3}-1, \cdots, d_{d_{1}+1}-1, d_{d_{1}+2}, \cdots, d_{n}\right)$ has a collapsible realization $H$ and assume that $v_{2}, v_{3}, \cdots, v_{d_{1}+1}$ have degree $d_{2}-1, d_{3}-1, \cdots, d_{d_{1}+1}-1$, respectively. Then we can get a realization $H^{\prime}$ of $d$ from $H$ by adding a new vertex $v_{1}$ and joining $v_{1}$ to $v_{2}, v_{3}, \cdots, v_{d_{1}+1}$, respectively. By Corollary 4.2.2(ii) $H^{\prime}$ is collapsible. Now we may assume that $d_{n}=3$. 
Case 1. If $d_{3}=3$, then by Lemma $4.2 .4,\left(d_{1}, d_{2}, 3, \cdots, 3\right)$ is collapsible.

Case 2. If $d_{3} \geq 4$, then $d_{1}-1 \geq d_{2}-1 \geq d_{3}-1 \geq 3$ and $d_{4} \geq \cdots \geq d_{n}=3$. By Lemma 4.2.6, $\left(d_{1}-1, d_{2}-1, d_{3}-1, d_{4}, \cdots, d_{n-1}\right)$ is graphic. By the induction hypothesis, $\left(d_{1}-1, d_{2}-1, d_{3}-1, d_{4}, \cdots, d_{n-1}\right)$ has a collapsible realization $K$ and assume that $u_{1}, u_{2}, u_{3}$ has degree $d_{1}-1, d_{2}-1, d_{3}-1$, respectively. Then we can get a realization $K^{\prime}$ of $d$ from $K$ by adding a new vertex $u$ and joining $u$ to $u_{1}, u_{2}, u_{3}$, respectively. By Corollary 4.2.2(ii) $K^{\prime}$ is collapsible.

\subsection{Supereulerian Sequence and Hamiltonian Line Graph}

Lemma 4.3.1 If $d=\left(d_{1}, d_{2}, \cdots, d_{n}\right)$ is a nonincreasing graphic sequence with $d_{n} \geq 2$, then there exists a 2-edge-connected realization of $d$.

Proof. Choose $G$ to be a realization of $d$ such that $G$ has as few components as possible. Therefore,the following claim holds.

Claim $2 G$ is connected.

Proof. Suppose, to the contrary, that $G$ has more than one components. Let $G_{1}, G_{2}$ be two components of $G$ and $e_{1}=u_{1} v_{1} \in E\left(G_{1}\right), e_{2}=u_{2} v_{2} \in E\left(G_{2}\right)$. Then $G-\left\{e_{1}, e_{2}\right\}+$ $\left\{u_{1} u_{2}, v_{1} v_{2}\right\}$ is a realization of $d$ with fewer components than $G$, contradicting the choice of $G$.

If $d_{1}=d_{2}=\cdots=d_{n}=2$, then $C_{n}$ is a 2-edge-connected realization of $d$. Now suppose that $d_{1}>2$. Then the following claim holds.

Claim 3 There is a a 2-edge-connected realization of $d$.

Choose $G$ to be a realization of $d$ with $\kappa^{\prime}(G)$ as large as possible. By Claim 2, $\kappa^{\prime}(G) \geq 1$. Suppose, to the contrary, that $\kappa^{\prime}(G)=1$ and furthermore, we can choose 
$G$ to be a realization of $d$ with as few cut edges as possible. Let $e=u v$ be a cut edge such that one of the component $G_{1}$ of $G-e$ is 2-edge-connected. Assume $u \in V\left(G_{1}\right)$. Then $d(u) \geq 3$. Suppose that $u v \cdots w$ is a path of $G$ such that the internal vertices on this path are of degree 2 and so $d(w) \geq 3$ (it is possible that $w=v$ ). Then there are $u u_{1}, u u_{2} \in E\left(G_{1}\right)$ and $w w_{1}, w w_{2} \in E\left(G_{2}\right)$. Now we can get $G^{\prime}$ from $G$ by deleting $u u_{1}, u u_{2}, w w_{1}, w w_{2}$ and adding $u_{1} w_{1}, u_{2} w_{2}$, and get $G^{\prime \prime}$ from $G^{\prime}$ by first dividing $u_{1} w_{1}$ into $u_{1} u^{\prime}$ and $u^{\prime} w_{1}$, dividing $u_{2} w_{2}$ into $u_{1} w^{\prime}$ and $w^{\prime} w_{2}$ and then identifying $u$ and $u^{\prime}, w$ and $w^{\prime}$. Then $G^{\prime \prime}$ is a realization of $d$ with fewer cut edges than $G$, contradicting the choice of $G$.

Lemma 4.3.2 If $d=\left(d_{1}, d_{2}, \cdots, d_{n}\right)$ is a nonincreasing sequence with $d_{1}=n-1, d_{n}=2$, then $d=\left(d_{1}, d_{2}, \cdots, d_{n}\right)$ is graphic if and only if $(i) d^{\prime}=\left(d_{1}-1, d_{2}-1, \cdots, d_{n-1}\right)$ is graphic when $d_{2} \geq n-2$ and (ii) $d^{\prime \prime}=\left(d_{1}-2, d_{2}, \cdots, d_{n-2}\right)$ is graphic or $d^{\prime \prime \prime}=$ $\left(d_{1}-1, d_{2}, \cdots, d_{i-1}, d_{i}-1, d_{i+1}, \cdots, d_{n-1}\right)$ for some $d_{i} \geq 3$ is graphic when $d_{2} \leq n-3$.

Proof. Let $d=\left(d_{1}, d_{2}, \cdots, d_{n}\right)$ be a nonincreasing sequence with $d_{1}=n-1, d_{n}=2$. Suppose that $d=\left(d_{1}, d_{2}, \cdots, d_{n}\right)$ is graphic. We consider the following three cases.

Case 1. $d_{2}=n-1$.

Let $G$ be a realization $d$ with $d\left(v_{i}\right)=d_{i}$ for $1 \leq i \leq n$. Since $d_{1}=d_{2}=n-1, v_{1} v_{n} \in$ $E(G)$ and $v_{2} v_{n} \in E(G)$ and so $G-v_{n}$ is a realization of $d^{\prime}=\left(d_{1}-1, d_{2}-1, \cdots, d_{n-1}\right)$.

Case 2. $d_{2}=n-2$.

In this case, the following claim holds.

Claim $4 d$ has a realization $G$ such that $N\left(v_{n}\right)=\left\{v_{1}, v_{2}\right\}$.

Proof Let $G$ be a realization of $d$. If $N\left(v_{n}\right)=\left\{v_{1}, v_{2}\right\}$, we are done. Otherwise, since $d\left(v_{1}\right)=n-1, N\left(v_{n}\right)=\left\{v_{1}, v_{i}\right\}$. Then $d\left(v_{i}\right) \leq n-2$ and there exists $v_{j}$ such that $v_{i} v_{j} \notin E(G)$. Since $d\left(v_{2}\right)=n-2$ and $v_{n} \notin N\left(v_{2}\right), v_{j} \neq v_{2}$ and $v_{j} \in N\left(v_{2}\right)$. So $G-\left\{v_{2} v_{j}, v_{i} v_{n}\right\}+\left\{v_{2} v_{n}, v_{i} v_{j}\right\}$ is a realization of $d$ with $N\left(v_{n}\right)=\left\{v_{1}, v_{2}\right\}$. 
Let $G$ be a realization of $d$ with $d\left(v_{i}\right)=d_{i}$ for $1 \leq i \leq n$ and $N\left(v_{n}\right)=\left\{v_{1}, v_{2}\right\}$. Then $G-v_{n}$ is a realization of $d^{\prime}=\left(d_{1}-1, d_{2}-1, \cdots, d_{n-1}\right)$.

Case 3. $d_{2} \leq n-3$.

In this case, there exists a realization $G$ of $d$ with $N\left(v_{n}\right)=\left\{v_{n-1}, v_{1}\right\}, N\left(v_{n-1}\right)=$ $\left\{v_{1}, v_{n}\right\}$ or $N\left(v_{n}\right)=\left\{v_{1}, v_{i}\right\}$ and $d\left(v_{i}\right) \geq 3$. In the former case, $G-\left\{v_{n}, v_{n-1}\right\}$ is a realization of $d^{\prime \prime}=\left(d_{1}-2, d_{2}, \cdots, d_{n-2}\right)$. In the latter case, $G-v_{n}$ is a realization of $d^{\prime \prime \prime}=\left(d_{1}-1, d_{2}, \cdots, d_{i-1}, d_{i}-1, d_{i+1}, \cdots, d_{n-1}\right)$.

Conversely, if $d^{\prime}=\left(d_{1}-1, d_{2}-1, \cdots, d_{n-1}\right)$ is graphic, then there is a realization $G^{\prime}$ of $d^{\prime}$ and so $G^{\prime}+\left\{v_{1} v_{n}, v_{2} v_{n}\right\}$ is a realization of $d$; if $d^{\prime \prime}=\left(d_{1}-2, d_{2}, \cdots, d_{n-2}\right)$ is graphic, then there is a realization $G^{\prime \prime}$ of $d^{\prime \prime}$ and so $G^{\prime \prime}+\left\{v_{1} v_{n}, v_{n} v_{n-1}, v_{n-1} v_{1}\right\}$ is a a realization of $d$; if $d^{\prime \prime \prime}=\left(d_{1}-1, d_{2}, \cdots, d_{i-1}, d_{i}-1, d_{i+1}, \cdots, d_{n-1}\right)$ is graphic, then there is a realization $G^{\prime \prime \prime}$ of $d^{\prime \prime \prime}$ and so $G^{\prime \prime \prime}+\left\{v_{1} v_{n}, v_{i} v_{n}\right\}$ is a realization of $d$.

Lemma 4.3.3 If $d=\left(d_{1}, d_{2}, \cdots, d_{n}\right)$ is a nonincreasing sequence with $d_{1} \leq n-2$ and $d_{n}=2$, then $d=\left(d_{1}, d_{2}, \cdots, d_{n}\right)$ is graphic if and only if $(i) d^{\prime}=\left(d_{1}, d_{2}, \cdots, d_{n-1}\right)$ is graphic or $d^{\prime \prime}=\left(d_{1}, d_{2}, \cdots, d_{i}-1, \cdots, d_{j}-1, \cdots, d_{n-1}\right)$ for some $d_{i} \geq 3$ and $d_{j} \geq 3$.

Proof. Let $d=\left(d_{1}, d_{2}, \cdots, d_{n}\right)$ be a nonincreasing sequence with $d_{1} \leq n-2$ and $d_{n}=$ 2. Suppose that $d=\left(d_{1}, d_{2}, \cdots, d_{n}\right)$ is graphic. Then there exists a 2-edge-connected realization $G$ of $d$ with $d\left(v_{i}\right)=d_{i}$ for $1 \leq i \leq n$. Suppose that $N\left(v_{n}\right)=\left\{v_{i}, v_{j}\right\}$. If $v_{i} v_{j} \notin E(G)$, then $G-v_{n}+\left\{v_{i} v_{j}\right\}$ is a realization of $\left(d_{1}, d_{2}, \cdots, d_{n-1}\right)$. Now suppose that $v_{i} v_{j} \in E(G)$ and we distinguish the following two cases:

Case 1: $\left\{v_{n}, v_{i}, v_{j}\right\} \cup N\left(v_{i}\right) \cup N\left(v_{j}\right) \neq V(G)$.

Let $T=V(G) \backslash\left(\left\{v_{n}, v_{i}, v_{j}\right\} \cup N\left(v_{i}\right) \cup N\left(v_{j}\right)\right)$. If there is $v_{s} \in T$ such that $N\left(v_{s}\right) \cap$ $\left(N\left(v_{i}\right) \triangle N\left(v_{j}\right)\right) \neq \emptyset$, then we assume that $v_{t} \in N\left(v_{s}\right) \cap\left(N\left(v_{i}\right) \backslash N\left(v_{j}\right)\right)$. Now we can get a realization $G^{\prime}$ of $d^{\prime}=\left(d_{1}, d_{2}, \cdots, d_{n-1}\right)$ from $G$ by deleting $v_{n}$, splitting $v_{s} v_{t}$ to $v_{s} v_{j^{\prime}}, v_{j^{\prime}} v_{i^{\prime}}, v_{i^{\prime}} v_{t}$ and then identifying $v_{i}$ and $v_{i^{\prime}}, v_{j}$ and $v_{j^{\prime}}$. Otherwise, for any vertex $v \in T, N(v) \cap\left(N\left(v_{i}\right) \triangle N\left(v_{j}\right)\right)=\emptyset$, which implies $N(T) \subseteq N\left(v_{i}\right) \cap N\left(v_{j}\right)$. Since $G$ is 2-edge-connected, then there are $v_{p}, v_{q} \in N\left(v_{i}\right) \cap N\left(v_{j}\right)$ (it is possible that $v_{p}=v_{q}$ ) and 
$v_{s}, v_{t} \in T$ (it is possible that $v_{s}=v_{t}$ ) such that $v_{s} v_{p}, v_{t} v_{q} \in E(G)$. Then we can get a realization $G^{\prime}$ of $\left(d_{1}, d_{2}, \cdots, d_{n-1}\right)$ from $G$ by deleting $v_{n}$, splitting $v_{s} v_{p}$ into $v_{s} v_{i^{\prime}}, v_{i^{\prime}} v_{p}$, splitting $v_{s} v_{q}$ into $v_{s} v_{j^{\prime}}, v_{j^{\prime} v_{q}}$ and then identifying $v_{i}$ and $v_{i^{\prime}}, v_{j}$ and $v_{j^{\prime}}$.

Case 2: $\left\{v_{n}, v_{i}, v_{j}\right\} \cup N\left(v_{i}\right) \cup N\left(v_{j}\right)=V(G)$.

In this case, $d_{i} \geq 3$ and $d_{j} \geq 3$. Otherwise, $\triangle(G)=n-1$, a contradiction. So $G-v_{n}$ is a realization of $d^{\prime \prime}=\left(d_{1}, d_{2}, \cdots, d_{i}-1, \cdots, d_{j}-1, \cdots, d_{n-1}\right)$.

Conversely, if $d^{\prime}=\left(d_{1}, d_{2}, \cdots, d_{n-1}\right)$ is graphic, then there is a realization $G^{\prime}$ of $d^{\prime}$ and so we can get a realization $G$ of $d$ by choosing an edge $e=v_{i} v_{j} \in E\left(G^{\prime}\right)$ and dividing it into $v_{i} v_{n}$ and $v_{n} v_{j}$. If $d^{\prime \prime}=\left(d_{1}, d_{2}, \cdots, d_{i}-1, \cdots, d_{j}-1, \cdots, d_{n-1}\right)$ is graphic, then there is a realization $G^{\prime \prime}$ of $d^{\prime \prime}$ and so we can get a realization $G$ of $d$ by adding vertex $v_{n}$ and edges $v_{i} v_{n}, v_{j} v_{n}$ to $G^{\prime \prime}$.

Theorem 4.3.4 If $d=\left(d_{1}, d_{2}, \cdots, d_{n}\right) \in \mathcal{G}$ is a nonincreasing sequences with $d_{n} \geq 2$, then $d$ has a supereulerian realization.

Proof of Theorem 4.1.2. By induction on $n$.

If $n=3$, then $(2,2,2) \in \mathcal{G}, K_{3}$ is supereulerian.

Suppose the theorem holds for all nonincreasing graphic degree sequences with fewer than $n$ entries. Let $d=\left(d_{1}, d_{2}, \cdots, d_{n}\right) \in \mathcal{G}$ be a nonincreasing sequences with $d_{n} \geq 2$. If $d_{n} \geq 3$, then by Theorem 4.2.7, $d$ has a collapsible realization $G$. By Corollary 4.2.2 (iii), $G$ is supereulerian. If $d_{1}=d_{2}=\cdots=d_{n}=2$, then $C_{n}$ is a supereulerian realization of $d$.

In the following, we assume that $d_{1}>d_{n}=2$. We consider two cases.

Case 1: $d_{1} \leq n-2$.

By Lemma 4.3.3, $d^{\prime}=\left(d_{1}, d_{2}, \cdots, d_{n-1}\right)$ is graphic or $d^{\prime \prime}=\left(d_{1}, d_{2}, \cdots, d_{i}-1, \cdots, d_{j}-\right.$ $\left.1, \cdots, d_{n-1}\right)$ with $d_{i} \geq 3$ and $d_{j} \geq 3$ is graphic. If $d^{\prime}$ is graphic, by the induction hypothesis, there is a supereulerian realization $G^{\prime}$ of $d^{\prime}$. Let $C^{\prime}$ be a spanning eulerian subgraph of $G^{\prime}$ and $e=u v$ be an edge of $C^{\prime}$. Then by splitting $e$ of $G^{\prime}$ into $u v_{n}, v_{n} v$, we get a supereulerian 
realization of $d$. If $d^{\prime \prime}$ with $d_{i} \geq 3$ and $d_{j} \geq 3$ is graphic, then by the induction hypothesis, there is a supereulerian realization $G^{\prime \prime}$ of $d^{\prime \prime}$. Let $C^{\prime \prime}$ be a spanning eulerian subgraph of $G^{\prime \prime}$. If $v_{i} v_{j} \in E\left(G^{\prime \prime}\right)$, then let $C_{1}=v_{i} v_{j} v_{n}$ and so $G=G^{\prime \prime}+\left\{v_{i} v_{n}, v_{j} v_{n}\right\}$ is a supereulerian realization of $d$. If $v_{i} v_{j} \notin E\left(G^{\prime \prime}\right)$, then we can get a realization $G$ of $d$ from $G^{\prime \prime}+\left\{v_{i} v_{j}\right\}$ by splitting an edge $e=u v$ of $C^{\prime}$ into $u v_{n}$ and $v_{n} v$.

Case 2: $d_{1}=n-1$.

By Lemma 4.3.2, $d^{\prime}=\left(d_{1}-1, d_{2}-1, \cdots, d_{n-1}\right)$ or $d^{\prime \prime}=\left(d_{1}-2, d_{2}, \cdots, d_{n-3}\right)$ or $d^{\prime \prime \prime}=\left(d_{1}-1, \cdots, d_{i-1}, d_{i}-1, d_{i+1}, \cdots, d_{n-1}\right)$ is graphic. If $d^{\prime}\left(\right.$ or $d^{\prime \prime}$ or $\left.d^{\prime \prime \prime}\right)$ is graphic, then by the inductive hypothesis, there is a supereulerian realization $G^{\prime}$ of $d^{\prime}$ (or $d^{\prime \prime}$ or $d^{\prime \prime \prime}$ ). Let $C^{\prime}$ be a spanning eulerian subgraph of $G^{\prime}$. Let $C_{1}=v_{n} v_{1} v_{2} v_{n}$ (or $C_{1}=v_{1} v_{n} v_{n-1} v_{1}$ or $\left.C_{1}=v_{1} v_{n} v_{i} v_{1}\right)$. Since $v_{1} v_{2} \in E\left(G^{\prime}\right)$ (or $v_{n-1}, v_{n} \notin V\left(G^{\prime}\right)$ or $v_{1} v_{i} \in E\left(G^{\prime}\right)$ ), $G^{\prime} \triangle C_{1}$ is a supereulerian realization of $d$ with a spanning eulerian subgraph $C^{\prime} \triangle C_{1}$.

Note that if $G$ is supereulerian, then $\delta(G) \geq 2$ and so $d_{n} \geq 2$, we have the following corollary.

Corollary 4.3.5 If $d=\left(d_{1}, d_{2}, \cdots, d_{n}\right) \in \mathcal{G}$ is a nonincreasing sequences, then $d$ has a supereulerian realization if and only if $d_{n} \geq 2$.

Theorem 4.3.6 (Harry and Nash-Williams, [11]) Let $|E(G)| \geq 3$. Then $L(G)$ is hamiltonian if and only if $G$ has a dominating eulerian subgraph.

Proof of Theorem 4.1.5. $(i) \Rightarrow(i i)$. Let $G$ be a realization of $d$ such that $L(G)$ is hamiltonian. By Theorem 4.3.6, $G$ has a dominating eulerian subgraph $H$. If $d_{1}=n-1$ and $\sum_{d_{i}=1} d_{i}>\sum_{d_{j} \geq 2}\left(d_{j}-2\right)$, then $G=K_{1, n-1}$. Assume that $G$ is not $K_{1, n-1}$. Then $H$ is nontrivial. For any $v_{i}$ with $d\left(v_{i}\right)=1, v_{i}$ must be adjacent to a vertex $v_{j}$ in $H$ and so $d_{G-E(H)}\left(v_{j}\right)$ is no less than the number of degree 1 vertices adjacent to $v_{j}$. Furthermore, since $H$ is eulerian and nontrivial, $d_{H}\left(v_{j}\right) \geq 2$ and so $\sum_{d_{i}=1} d_{i} \leq \sum_{d_{j} \geq 2}\left(d_{j}-2\right)$.

(ii) $\Rightarrow$ (iii) Suppose $d \in \mathcal{G}$ is a nonincreasing sequence such that $d_{n} \geq 1$ and $\sum_{d_{i}=1} d_{i} \leq \sum_{d_{j} \geq 2}\left(d_{j}-2\right)$. If $d_{n} \geq 2$, then by Theorem 4.3.4, $d$ has a supereulerian realization. So we assume that $d_{n}=1$. 
Claim 5 Any realization of d contains a cycle.

Suppose that there exists a realization $G$ of $d$ such that $G$ is a tree. We may assume that $d_{i} \geq 2$ for $1 \leq i \leq k$ and $d_{j}=1$ for $k+1 \leq j \leq n$. Then

$$
\sum_{i=1}^{k} d_{i}+(n-k)=\sum_{i=1}^{k} d_{i}+\sum_{i=k+1}^{n} d_{i}=\sum_{i=1}^{n} d_{i}=2|E(G)|=2(n-1),
$$

and so

$$
\sum_{i=1}^{k}\left(d_{i}-2\right)+(n-k)=2(n-1)-2 k \text {. }
$$

Hence

$$
\sum_{d_{j} \geq 2}\left(d_{j}-2\right)=\sum_{i=1}^{k}\left(d_{i}-2\right)=2(n-1)-2 k-(n-k)=n-k-2<n-k=\sum_{d_{i}=1} d_{i}
$$

contrary to (4.1). This completes Claim 5 and we assume $G$ is a realization of $d$ containing a nontrivial cycle $C$.

Claim 6 There is a realization $G$ of $d$ such that $\delta\left(G-D_{1}(G)\right) \geq 2$.

As $G$ contains a nontrivial cycle $C, G-D_{1}(G)$ is not empty. Let $S=N\left(D_{1}(G)\right)$. It suffices to show that for each $s \in S, N_{G-D_{1}(G)}(s) \geq 2$. Suppose, to the contrary, that there is $s \in S$ such that $N_{G-D_{1}(G)}(s)=2$. Choose $G$ to be a graph such that the elements in $P(G)=\left\{s: s \in S\right.$ with $d_{G}(s)=d_{t} \geq 2$ such that $\left.N_{G-D_{1}(G)}(s)=1\right\}$ is as few as possible. Let $x \in P(G)$. Then $x \notin C$. Choose $e \in E(C)$ and we subdivide $e$ and let $v_{e}$ denote the subdivision vertex. And we delete $d_{t}-1$ pendent edges of $x$, add $d_{t}-2$ pendent edges to $v_{e}$ and denote the resulting graph $G_{x}$ ( Note that if $d_{t}-2=0$, then we subdivide $e$ without adding any pendent edges). So $d_{G_{x}}\left(v_{e}\right)=2+d_{t}-2=d_{t}$ and $\left|D_{1}\left(G_{x}\right)\right|=\left|\left(D_{1}(G)-N_{1}(x)\right) \cup\{x\}\right|+d_{t}-2=\left|D_{1}(G)\right|-\left(d_{t}-1\right)+1+d_{t}-2=\left|D_{1}(G)\right|$ but $\left|P\left(G_{x}\right)\right|<|P(G)|$, contradicting the choice of $G$.

$($ iii $) \Rightarrow(i)$ If $G$ is a realization of $d$ such that $\delta\left(G-D_{1}(G)\right)$ is supererulerian, then by Theorem 4.3.6, $L(G)$ is hamiltonian. 


\section{Chapter 5}

\section{Regular Matroids without Disjoint Circuits}

\subsection{The Problem and the Main Results}

If $G$ is a graph and if $V_{1}, V_{2}$ are two disjoint vertex subsets of $G$, then $\left[V_{1}, V_{2}\right]$ denote the set of edges in $G$ with one end in $V_{1}$ and the other end in $V_{2}$. For a vertex $v \in V(G)$, let

$$
E_{G}(v)=\{e \in E(G): e \text { is incident with } v\} .
$$

Let $M$ and $N$ denote two matroids. If $\{e, f\}$ is a circuit of $M^{*}$ and if $M / f=N$, then $M$ is a serial extension of $N$. In this case, we say that $f$ is serial to $e$. Note that being serial is an equivalence relation on $E(M)$ for a matroid $M$. The corresponding equivalence classes are the serial classes of $M$. Dually, two elements $e, f$ are parallel in $M$ if they are serial in $M^{*}$; being parallel is an equivalence relation on $E(M)$ and the equivalence classes are the parallel classes of $M$. An equivalence class is nontrivial if it has more than one elements.

In 1960, Erdös and Pósa consider the problem of determining all connected graphs that do not have edge-disjoint circuits. We view the complete graph $K_{3}$ as a plane graph 
and let $K_{3}^{*}$ denote the geometric dual of the plane graph $K_{3}$.

Theorem 5.1.1 (Erdös and Pósa [8], also see Theorem 3.1, Theorem 3.2 of Bollobás [1]) Let $G$ be a graph with $\delta(G) \geq 3$. The following are equivalent.

(i) $G$ does not have edge-disjoint circuits.

(ii) $G \in\left\{K_{3,3}, K_{3}^{*}, K_{4}\right\}$.

Since a graph $G$ does not have disjoint circuits if and only if any subdivision of $G$ does not have disjoint circuits, the following corollary follows immediately.

Corollary 5.1.2 (Erdös and Pósa [8], also see Corollary 3.3 of Bollobás [1]) Let G be a simple graph of order $n \geq 3$.

(i) If $|E(G)| \geq n+4$, then $G$ has 2 edge-disjoint circuits.

(ii) The graph $G$ with $|E(G)|=n+3$ does not have edge-disjoint circuits if and only if $G$ can be obtained from a subdivision $G_{0}$ of $K_{3,3}$ by adding a forest and exactly one edge, joining each tree of the forest to $G_{0}$.

Theorem 5.1.1 can be viewed as a result on cosimple graphic matroids. Thus we consider generalizing Theorem 5.1.1 to matroids. Our main results of this note are the following.

Theorem 5.1.3 Let $M$ be a connected cosimple regular matroid. The following are equivalent.

(i) $M$ does not have disjoint circuits.

(ii) $M \in\left\{M\left(K_{3,3}\right)\right\} \cup\left\{M^{*}\left(K_{n}\right), n \geq 3\right\}$.

Corollary 5.1.4 Let $M$ be a regular matroid. Then $M$ has no disjoint circuits if and only if one of the following holds:

(i) $M=U_{m, m}$, for some integer $m>0$, or

(ii) $M$ is a serial extension of a member in $\left\{M\left(K_{3,3}\right), U_{0,1}\right\} \cup\left\{M^{*}\left(K_{n}\right), n \geq 3\right\}$, or

(iii) $M=M_{1} \bigoplus M_{2}$ is the direct sum of two matroids $M_{1}$ and $M_{2}$, where $M_{1}$ is a serial extension of a member in $\left\{M\left(K_{3,3}\right), U_{0,1}\right\} \cup\left\{M^{*}\left(K_{n}\right), n \geq 3\right\}$ and where $M_{2} \cong U_{m, m}$, for some $m=|E(M)|-\left|E\left(M_{1}\right)\right| \geq 1$. 


\subsection{Proof of the Main Results}

We follow Seymour [21] to introduce the notion of binary matroid sums. Given two sets $X$ and $Y$, the symmetric difference of $X$ and $Y$, is

$$
X \Delta Y=(X \cup Y)-(X \cap Y)
$$

Let $M_{1}$ and $M_{2}$ be two binary matroids where $E\left(M_{1}\right)$ and $E\left(M_{2}\right)$ may intersect. Define $M_{1} \Delta M_{2}$ to be the binary matroid on $E=E\left(M_{1}\right) \Delta E\left(M_{2}\right)$ whose cycles are all subsets of $E$ of the form $C_{1} \Delta C_{2}$, where $C_{1}$ is a cycle of $M_{1}$ and $C_{2}$ is a cycle of $M_{2}$. The binary matroid sums are defined as follows.

(i) If $E\left(M_{1}\right) \cap E\left(M_{2}\right)=\emptyset$, then $M_{1} \Delta M_{2}$ is the 1-sum of $M_{1}$ and $M_{2}$ (also referred as a direct sum).

(ii) If $E\left(M_{1}\right) \cap E\left(M_{2}\right)=\left\{e_{0}\right\}$, such that, for each $i \in\{1,2\}$, the element $e_{0}$ is neither a loop nor a coloop of $M_{i}$, then $M_{1} \Delta M_{2}$ is the 2-sum of $M_{1}$ and $M_{2}$.

(iii) If $E\left(M_{1}\right) \cap E\left(M_{2}\right)=C$, where $C$ is a 3 -circuit of both $M_{1}$ and $M_{2}$, such that $C$ includes no cocircuit of either $M_{1}$ or $M_{2}$, and such that for $i \in\{1,2\},\left|E\left(M_{i}\right)\right| \geq 7$, then $M_{1} \Delta M_{2}$ is the 3-sum of $M_{1}$ and $M_{2}$.

For $k=1,2,3$, we also use $M_{1} \bigoplus_{k} M_{2}$ to denote the $k$-sum of two matroids $M_{1}$ and $M_{2}$. If each of $M_{1}$ and $M_{2}$ is isomorphic to a proper minor of $M_{1} \bigoplus_{k} M_{2}$, then we say that $M$ is a proper $k$-sum of $M_{1}$ and $M_{2}$. For the case $\mathrm{k}=1$, we also use $M_{1} \bigoplus M_{2}$ for $M_{1} \bigoplus_{1}$ to denote the direct sum of $M_{1}$ and $M_{2}$.

Let $A$ denote the matrix below

$$
A=\left[\begin{array}{llllllllll}
1 & 0 & 0 & 0 & 0 & 1 & 1 & 0 & 0 & 1 \\
0 & 1 & 0 & 0 & 0 & 1 & 1 & 1 & 0 & 0 \\
0 & 0 & 1 & 0 & 0 & 0 & 1 & 1 & 1 & 0 \\
0 & 0 & 0 & 1 & 0 & 0 & 0 & 1 & 1 & 1 \\
0 & 0 & 0 & 0 & 1 & 1 & 0 & 0 & 1 & 1
\end{array}\right]
$$

and let $R_{10}$ denote the binary matroid $M_{2}[A]$.

Seymour's regular matroid decomposition theorem can be applied to cosimple matroids in the following form. 
Theorem 5.2.1 (Seymour [20]) Let $M$ be a cosimple connected regular matroid. Then one of the following holds.

(i) $M$ is cosimple and graphic.

(ii) $M$ is cosimple and cographic.

(iii) $M$ is isomorphic to $R_{10}$.

(iv) For $i \in\{2,3\}, M=M_{1} \bigoplus_{k} M_{2}$ is the proper 2-sum or 3-sum of two cosimple regular matroids $M_{1}$ and $M_{2}$, where both $M_{1}$ and $M_{2}$ are isomorphic to proper minors of $M$.

The following lemma is straightforward.

Lemma 5.2.2 Let $G$ be a graph. If $M(G)$ is cosimple, then $\delta(G) \geq 3$.

Proof: Note that any edge incident with a degree 1 vertex in $G$ must be a loop of $M^{*}(G)$, and that the edges incident with a degree 2 vertex in $G$ must be in a parallel class of $M^{*}(G)$. Since $M(G)$ is cosimple, $M^{*}(G)$ does not have loops or nontrivial parallel classes. Hence we must have $\delta(G) \geq 3$.

Proof of Theorem 5.1.3 We first show that Theorem 5.1.3(i) implies Theorem 5.1.3(ii), and so we assume the $M$ is a connected cosimple regular matroid with no disjoint circuits. By Theorem 5.2.1, one of the conclusions in Theorem 5.2.1 must hold.

If $M$ is graphic, then we may assume that for some connected graph $G, M=M(G)$. By Lemma 5.2.2, $\delta(G) \geq 3$. Since $G$ has no disjoint circuits, by Theorem 5.1.1, $G \in$ $\left\{K_{3,3}, K_{3}^{*}, K_{4}\right\}$, and so Theorem 5.1.3(ii) holds.

If $M$ is cographic, then we may assume that for some graph $G, M=M^{*}(G)$, where $G$ is a connected graph with $n=r(M)+1$ vertices. Since $M$ is cosimple, $G$ is a simple graph, and so $G$ is a spanning subgraph of $K_{n}$, the complete graph on $n$ vertices. Let $V(G)=\left\{v_{1}, v_{2}, \cdots v_{n}\right\}$. If $G \neq K_{n}$, then we may assume that $v_{1} v_{2} \notin E(G)$. In this case, $E_{G}\left(v_{1}\right) \cap E_{G}\left(v_{2}\right)=\emptyset$, contrary to Theorem 5.1.3(i). Therefore, we must have $G=K_{n}$, and so $M \in\left\{M^{*}\left(K_{n}\right), n \geq 3\right\}$.

If $M$ is isomorphic to $R_{10}$, then it is well known that $R_{10}$ is a disjoint union of a 4-circuit and a 6-circuit, contrary to Theorem 5.1.3(i). Thus $M \cong R_{10}$ is impossible. 
Now suppose that Theorem 5.2.1(iv) holds. We argue by induction on $|E(M)|$. Since any matroid with at most 3 elements must be graphic, we assume that $|E(M)|=n \geq$ 4, and Theorem 5.2.1(ii) holds for any matroid $M$ satisfying Theorem 5.1.3(i) with $|E(M)|<n$.

Since Theorem 5.2.1(iv) holds, for some $i \in\{2,3\}, M=M_{1} \bigoplus_{i} M_{2}$ is the proper $i$-sum of two cosimple regular matroids $M_{1}$ and $M_{2}$, where both $M_{1}$ and $M_{2}$ are proper minors of $M$.

If one of $M_{1}$ or $M_{2}$ has two disjoint circuits, then by the definition of binary matroid sums, $M$ would also have disjoint circuits, contrary to Theorem 5.1.3(i). Therefore, for each $i, M_{i}$ does not have disjoint circuits. Since $M_{i}$ is a proper minor of $M$, by induction, $M_{1}, M_{2} \in\left\{M\left(K_{3,3}\right)\right\} \cup\left\{M^{*}\left(K_{n}\right), n \geq 3\right\}$.

If $i=2$, then we may assume that $e_{0} \in E\left(M_{1}\right) \cap E\left(M_{2}\right)$. By the definition of 2sum and by the fact that $M_{1}, M_{2} \in\left\{M\left(K_{3,3}\right)\right\} \cup\left\{M^{*}\left(K_{n}\right), n \geq 3\right\}, \exists C_{1} \in \mathcal{C}\left(M_{1}\right)$ and $C_{2} \in \mathcal{C}\left(M_{2}\right)$ such that $e_{0} \notin C_{i}$. It follows that $C_{1} \cap C_{2}=\emptyset$ and so Theorem 5.1.3(i) is violated. Thus this is impossible.

Now assume that $i=3$, and $Z=E\left(M_{1}\right) \cap E\left(M_{2}\right)$ is a 3 element circuit of both $M_{1}$ and $M_{2}$. Recall that $M_{1}, M_{2} \in\left\{M\left(K_{3,3}\right)\right\} \cup\left\{M^{*}\left(K_{n}\right), n \geq 3\right\}$. By the definition of a 3 -sum, for any $i \in\{1,2\},\left|E\left(M_{i}\right)\right| \geq 7$ and so $M_{i} \notin\left\{M^{*}\left(K_{3}\right), M^{*}\left(K_{4}\right)\right\}$. Since there is no 3-circuits in either $M\left(K_{3,3}\right)$ or a $M^{*}\left(K_{n}\right)$ with $n>4$, it is impossible that both $|Z|=3$ and $Z \in \mathcal{C}\left(M_{1}\right) \cap \mathcal{C}\left(M_{2}\right)$. This contradiction shows that this case is also impossible.

Thus if Theorem 5.1.3(i) holds, then we must have $M \in\left\{M\left(K_{3,3}\right)\right\} \cup\left\{M^{*}\left(K_{n}\right), n \geq\right.$ $3\}$.

Conversely, suppose $M \in\left\{M\left(K_{3,3}\right)\right\} \cup\left\{M^{*}\left(K_{n}\right), n \geq 3\right\}$. Since $K_{3,3}$ is a bipartite simple graph, any circuit of $K_{3,3}$ has length at least 4 . Suppose that $K_{3,3}$ has two disjoint circuits $C_{1}$ and $C_{2}$, then since $K_{3,3}$ is 3-regular, we must have $V\left(C_{1}\right) \cap V\left(C_{2}\right)=\emptyset$, and so $6=\left|V\left(K_{3,3}\right)\right| \geq\left|V\left(C_{1}\right)\right|+\left|V\left(C_{2}\right)\right| \geq 8$, a contradiction. Hence $M\left(K_{3,3}\right)$ cannot have disjoint circuits. Suppose that $M=M^{*}\left(K_{n}\right), n \geq 3$ and write $V\left(K_{n}\right)=\left\{v_{1}, v_{2}, \cdots, v_{n}\right\}$. Suppose that $C_{1}$ and $C_{2}$ are two circuits of $M^{*}\left(K_{n}\right)$. Then $C_{1}$ is an edge cut of $K_{n}$ and so $C_{1}=\left[V_{1}, V_{2}\right]$, for some proper vertex subset $V_{1} \subseteq V(G)$ and $V_{2}=V(G)-V_{1}$. 
Similarly, $C_{2}=\left[W_{1}, W_{2}\right]$, where $\emptyset \neq W_{1} \subseteq V(G)$ and $W_{2}=V(G)-W_{1} \neq \emptyset$. We may assume that $v_{1} \in V_{1} \cap W_{1}$. If $V_{2} \cap W_{2} \neq \emptyset$, say $v_{2} \in V_{2} \cap W_{2}$, then $v_{1} v_{2} \in C_{1} \cap C_{2}$. If $V_{2} \cap W_{2}=\emptyset$, then we have $W_{2} \subseteq V_{1}, V_{2} \subseteq W_{1}$. Since $\emptyset \neq\left[V_{2}, W_{2}\right] \subseteq\left[V_{2}, V_{1}\right]=C_{1}$ and $\emptyset \neq\left[V_{2}, W_{2}\right] \subseteq\left[W_{1}, W_{2}\right]=C_{2}$, then $C_{1} \cap C_{2} \neq \emptyset$. This proves that $M^{*}\left(K_{n}\right)$ does not have disjoint circuits.

Proof of Corollary 5.1.4 It suffices to show, by induction on $|E(M)|$, that if $M$ has no disjoint circuits, then one of (i), (ii) and (iii) holds. Let $M$ be a regular matroid that does not have disjoint circuits.

We first assume that $M$ is connected. If $M$ has a loop or a coloop, then since $M$ is connected, we must have $M \in\left\{U_{0,1}, U_{1,1}\right\}$, and so Corollary 5.1.4 (i) or (ii) must hold. Thus we assume that $M$ is loopless and coloopless.

If $M$ is connected and cosimple, then by Theorem 5.1.3, $M$ is a member of $\left\{M\left(K_{3,3}\right)\right\} \cup$ $\left\{M^{*}\left(K_{n}\right), n \geq 3\right\}$ and so Corollary 5.1.4(ii) holds. Otherwise, $M$ has nontrivial serial classes. Let $\left\{e_{1}, e_{2}\right\}$ be a pair of serial elements in $M$. Since the intersection of any circuit and any cocircuit in a matroid $M$ cannot have exactly one element, any circuit in $M$ containing $e_{1}$ must also contain $e_{2}$. This implies that $M$ has no disjoint circuits if and only if $M / e_{2}$ has no disjoint circuits. By induction, $M / e_{2}$ is a serial extension of a member in $\left\{M\left(K_{3,3}\right), U_{0,1}\right\} \cup\left\{M^{*}\left(K_{n}\right), n \geq 3\right\}$. Since $M$ is a serial extension of $M / e_{2}, M$ is also a serial extension of a member in $\left\{M\left(K_{3,3}\right), U_{0,1}\right\} \cup\left\{M^{*}\left(K_{n}\right), n \geq 3\right\}$.

Now suppose that $M$ is not connected. Then $M=M_{1} \oplus M_{2} \bigoplus \cdots \bigoplus M_{k}$, where $M_{1}, M_{2}, \cdots, M_{k}$ are connected components of $M$. If $\forall i, M_{i}$ contains no circuits, then Corollary 5.1.4(i) holds. Otherwise, since $M$ has no disjoint circuits, exactly one connected component, say $M_{1}$, has at least one circuit. It follows that $M_{2} \bigoplus \cdots \bigoplus M_{k} \cong U_{n, n}$ and so Corollary 5.1 .4 (iii) must hold. 


\section{Bibliography}

[1] B. Bollobás, Extremal Graph Theory, Academic Press Inc., London, (1978).

[2] J. A. Bondy and U. S. R. Murty, Graph theory with applications, Macmillan, London and Elsevier, New York, 1976.

[3] P. A. Catlin, A reduction method to find spanning eulerian subgraphs, J. Graph Theory 12 (1988) 29-45.

[4] G. Chen, Hamiltonian graphs involving neighborhood intersections, Disc. Math. 112 (1993), 253-258 .

[5] G. Chen, Y. Egawa, X. Liu, and Saito, Essential independent set and Hamiltonian cycles, J. Graph Theory 21 (1996), 243-250.

[6] G. A. Dirc, Some theorems on abstract graphs, Proc. London Math. Soc. 2 (1952), 69-81.

[7] M. H. ElZahar, On circuits in gaphs, Disc. Math. 50 (1984), 227-230.

[8] P. Erdös and L. Pósa, On independent circuits contained in a graph, Canad. J. Math. (1965), 347-352.

[9] G. Fan, New sufficient conditions for cycles in graphs, J. Combin. Theory Ser. B 37 (1984), 221-227.

[10] R. J. Gould, Advances on the Hamiltonian problem- A survey, Graphs and Combinatorics 19 (2003), 7-52. 
[11] F. Harary and C. St. J. A. Nash-Williams, On eulerian and hamiltonian graphs and line graphs, Canad. Math. Bull. 8 (1965), 701-709.

[12] F. Jaeger, On interval hypergraphs and nowhere-zero flow in graphs, PResearch Report of Mathematics Aplication and Information, Universite Scientifique et Medicale et Institut National Polytechnique de Grenoble, No. 126, Juillet (1978).

[13] F. Jaeger, A note on subeulerian graphs, J. Graph Theory 3 (1979) 91-93.

[14] K. Zhao, H.-J. Lai and Y. Shao, New sufficient condition for hamiltonian graphs, Applied Math. Letters, to appear.

[15] K. Zhao, H.-J. Lai and J. Zhou, Hamiltonian-connected graphs with large neighborhoods and degrees, in submission.

[16] M. M. Matthews and D. P. Sumner, Hamiltonian results in $K_{1,3}$-free graphs, J. Graph Theory, 8( 1984) 139-146.

[17] O. Ore, Note on Hamiltonian circuits, Amer. Math. Monthly 67 (1960), 55.

[18] J. G. Oxley, Matroid Theory, Oxford University Press, New York, 1992.

[19] Z. Ryjáček, On a closure concept in claw-free graphs, J. Combin. Theory Ser. B, 70 (1997), 217-224.

[20] P. D. Seymour, Decomposition of regular matroids J. Combin. Theory Ser. B 28 (1980), 305-359.

[21] P. D. Seymour, Matroids and multicommodity flows, European J. Combin. Theory Ser. B. 2 (1981), 257-290.

[22] Y. Shao, Claw-Free Graphs and Line Graphs, Ph. D. Dissertation, West Virginia University, (2005).

[23] C. Thomassen, Reflections on graph theory, J. Graph Theory, 10 (1986), 309-324.

[24] D. J. A. Welsh, Matroid Theory, Academic Press, London, (1976). 
[25] S. M. Zhan, On hamiltonian line graphs and connectivity, Discrete Math. 89 (1991) 89-95.

[26] R. Luo, W. A. Zang and C-Q Zhang, Nowhere-zero 4-flows, simultaneous edgecolorings, and critical partial latin squares, Combinatorica 24 (4) (2004), 641-657. 